\title{
Transparency and Control in Platforms for Networked Markets
}

\author{
John Pang ${ }^{1}$, Weixuan $\mathrm{Lin}^{2}$, Hu Fu ${ }^{3}$, Jack Kleeman ${ }^{4}$, Eilyan Bitar ${ }^{2}$, Adam Wierman ${ }^{1}$ \\ ${ }^{1}$ California Institute of Technology, Pasadena, California, USA \\ ${ }^{2}$ Cornell University, Ithaca, New York, USA \\ ${ }^{3}$ University of British Columbia, Vancouver, British Columbia, Canada \\ ${ }^{4}$ University of Cambridge, Cambridge, UK
}

\begin{abstract}
In this work, we analyze the worst case efficiency loss of online platform designs under a networked Cournot competition model. Inspired by some of the largest platforms today, the platform designs considered tradeoffs between transparency and control, namely, (i) open access, (ii) controlled allocation and (iii) discriminatory access. Our results show that open access designs incentivize increased production towards perfectly competitive levels and limit efficiency loss, while controlled allocation designs lead to producer-platform incentive misalignment, resulting in low participation and unbounded efficiency loss. We also show that discriminatory access designs seek a balance between transparency and control, and achieve the best of both worlds, maintaining high participation rates while limiting efficiency loss. We also study a model of consumer search cost which further distinguishes between the three designs.
\end{abstract}

\section{Introduction}

The emergence of platforms has led to a new paradigm of online marketplaces. Platforms, unlike traditional retailers or providers, often do not produce or store physical goods, but instead matches producers to consumers and help set prices algorithmically. For example, a ride-sharing platform like Uber does not employ any of their drivers but instead aims to match drivers to riders and price these rides. Besides Uber, many other platforms connect producers to consumers in new and innovative ways to improve both provision of supply and fulfillment of demand, which leads to enormous efficiency gains and explosive growth. The estimated market value of some of these platforms alone is $\$ 4.3$ trillion US dollars as shown in [33, representing a significant proportion of the $\$ 56.8$ trillion US dollar market value on the top 2000 companies globally according to a [35] report.

There are multiple factors to consider in designing a successful platform, including matching, access control and pricing. There is an increasingly large literature on platform and two-sided market design, driven by the desire to understand what makes them successful. In a seminal work to understand two-sided markets, [66] compares the outcome from different matching structure organizations, e.g., profit-maximizing versus not-for-profit organizations. [9] then compares in a similar two-sided market model three different access control platform designs: monopolistic platforms, competing platforms where users can only choose one platform, and bottleneck platforms, where one side can choose to be on multiple platforms. More recently, [5] has shown that dynamic 
matching can improve control over the number of providers in networked market settings by utilizing information on agent's departure. Algorithmic pricing and its effect in real-world markets have also been actively studied in recent years, e.g., in [21,22,51],. Lately, more data-driven works, e.g., 29], have focused on balancing trade-offs between conflicting designs, e.g., guiding consumers to their most desired product while also strengthening sellers' incentives to offer low prices.

The focus of this paper is on access and allocation control designs with regards to tradeoffs between transparency and control. Platforms have very diverse access and allocation control designs even within the same industry. For example, within ride-sharing platforms, Didi requires a confirmation of pick-up and drop-off locations before allowing drivers to pick rides in an open access design while Uber assigns or allocates each ride to the nearest driver. More broadly, marketplaces like eBay have an open access design and seek to lower producer entry cost and increase competition, but may end up suffering from high consumer search cost. On the other hand, Amazon combines the scale of a populated marketplace with carefully differentiated access through their Buybox designs in a selective or discriminatory access design.

There are a variety of important open questions with regards to the design of access and allocation control. Firstly, what is the worst case efficiency loss of platforms under these designs? Secondly, what is the impact of allocation control, and are there any unintended incentives for strategic behavior that might decrease market efficiency? Lastly, is there a sweet spot between open access and controlling allocations that balances transparency and control, and limits efficiency loss?

\subsection{Our Contributions}

In this work, we model and analyze three different platform designs, (i) open access, (ii) controlled allocation and (iii) discriminatory access designs, and provide worst case efficiency loss bounds for each of them. We study these designs using the model of networked Cournot competition. Our analysis provides new results on efficiency loss, and illustrates the tradeoff between transparency and control in the design of platforms.

The first platform design we consider is the open access design (Section 31), exemplified by online marketplaces like eBay and Etsy. The advantages of an open access platform design are its fairness, transparency and low producer entry cost, and the "increased market competitiveness" driven by such attributes. This design also increases competition among producers, which usually leads to large sales volume and lower prices for consumers. We show that under the networked Cournot competition model, open access platform designs preserve a large proportion of the optimal demand fulfilled in each market, which plays a large role in limiting efficiency loss in the system. We prove a $2 / 3$ worst case guarantee on social welfare retention relative to the optimal social welfare under open access platform designs (Theorem 3.1). An integral piece of that result is a novel argument that the convex cost functions that maximize efficiency loss are linear cost functions, a generalization of the results from [46] for single market Cournot competition. This fact also allows for us to obtain worst case efficiency loss bounds as a function of the degree of asymmetry in producers' cost functions. Together, these results show that open access promotes sales volume, has a small efficiency loss when firms have symmetric cost, and retains a large proportion of the system's optimal social welfare.

Given the efficiency loss in open access designs, one may intuitively posit that the efficiency loss in open access platforms is due to poor allocation of supply to consumers. This is one of the many motivating factors towards considering a controlled allocation platform (Section 4). These 
platforms control the allocation over each individual transaction, in order to carefully allocate supply from all producers to all consumers. Many modern platforms use designs of these forms, e.g., drivers in ride-sharing platforms like Uber decide when to drive but not who to fetch. Given the prominence of these designs, one might expect that such platform designs are efficient. In contrast, we show that the worst case efficiency loss can grow linearly in the number of markets even when the controlled allocation platform optimizes for social welfare (Theorem 4.1). This highlights that controlled allocation platforms may end up being inefficient in spite of its good intentions. Additionally, we show that the controlled allocation platform under the networked Cournot competition model results in a networked Stackelberg game, where neither the existence nor uniqueness of Nash equilibrium can be guaranteed. We conclude Section 4 with a general result (Theorem 4.2) on the worst case efficiency loss under different choices of the market clearing mechanism. Specifically, we consider the setting in which the platform is allowed to choose its market clearing mechanism via the choice of its objective function used in market clearing, and show that any objective function that is a convex combination of consumer surplus and social welfare has the same unbounded worst case efficiency loss result while allocations that are more aligned to individual producers (such as revenue maximizing allocations) result in a smaller worst case efficiency loss.

The efficiency loss under controlled allocation designs suggests that the platform's allocation incentivizes decreasing production levels to a point where the optimal allocation of produced good cannot offset the efficiency loss associated with reduced production. On the other hand, the result on incentivizing production in open access platforms motivates us to carefully restrict producerconsumer pairs in a way that strikes a balance between transparency and control to improve the allocation while maintaining large proportions of demand fulfilled. Such a design is similar to what Amazon's Buybox attempts to do by first presenting a seller determined by Amazon based on various performance measures, and then presenting the possibility of looking at all possible sellers.

Motivated by this, we propose a new platform design called discriminatory access which optimizes over the edge set to increase the social welfare at the Nash equilibrium of the resulting networked Cournot competition. We show (Theorem 5.1) that while the guaranteed preserved proportion of optimal demand fulfilled may not be as large as the transparent open access platforms, the improved allocations as a result of controlling the network allows for an improved 3/4 worst case guarantee on social welfare retention for discriminatory access platforms. Similar to the open access platform results, we further explore the impact of cost asymmetry on the worst case efficiency loss. Specifically, when the production cost functions of firms are close to identical, it would be desirable to choose an open access design which maximizes the extent of competition between firms; on the other hand, if firms have substantially different cost functions, it would be desirable to choose a discriminatory access design, in which the platform picks out those firms with low production costs. A careful design over the connections in the discriminatory access platform is also often not trivial, and we show that the optimal network design problem can be written as a mathematical program with equilibrium constraints, and can be difficult to solve both analytically and computationally. However, under the restriction to linear cost functions - which were shown previously to yield the largest loss of efficiency in open access platforms - we propose and prove the optimality of a greedy algorithm that yields an optimal worst case network design (Theorem 5.2).

One factor for platform design that we have ignored to this point is search cost. Search costs are one form of consumer transaction or switching cost involved in searching for better alternatives. One of the initial goals of platforms is to lower search costs for consumers and lower entry costs 
for producers. Without careful understanding of the trade-offs between the aforementioned objectives and carefully balancing them, one can end up in a situation where search costs overwhelm consumers. For example, an open access platform design for a ride-sharing platform may not be a good idea, since the number of producers (in this case, drivers) in such platforms is large, resulting in large search costs for consumers. On the other hand, successful ride-sharing platforms, e.g., Uber, show only prices and do not offer alternative producers, eliminating any search cost involved in a transaction. To understand the impact of search costs on the efficiency of the three platform designs, we introduce a simple search cost model in Section 6 that yields important contrasts. In particular, under large consumer search cost, the worst case efficiency loss of open access platforms may no longer be bounded since welfare losses from search costs may outweigh the benefits of open access. On the other hand, the discriminatory access platform design remains efficient in the face of search costs and can be further optimized to balance search costs with efficiency of the matching.

\subsection{Related Work}

The proliferation of platforms and networked competition has led to a large and growing literature that covers many aspects of platform design, i.e., how to design successful platforms and why are some platforms more successful than others. Additionally, with more platforms collecting data, much of the recent work uses data to learn particular features in the successful design of platforms. In this paper, we present a study of the worst case efficiency loss of different designs with regards to access and allocation control for platforms under a networked Cournot model. Our work builds and relies on two major research fields - two-sided platform markets and networked competition.

\section{Two-sided Platform Markets.}

The recent growth of online platforms has led researchers to focus on identifying design features common to successful platforms. Earlier works in this area started by introducing different models of two-sided platform markets, e.g., [32] modeled the cross-network externalities between the two or more groups while the renowned work of [66] considered a model whereby pricing structure affects volume of transaction. They posit that price structure matters through (i) transaction costs existing between different sides, and (ii) constraints on types of transaction costs imposed by the platform. Other definitions and models are found in many other work, such as [39,68], and various platform designs are suggested in other works. We focus first on access and allocation control.

Open access is touted in [18] to increase competition among participants and shown in 65, 70, to be often designed to cope with issues such as fairness and openness. The classical example studied for open access is the online marketplace eBay studied in [27], which has been shown in [37, 43] to depend much on reputation and regulation. Lastly, 41] shows that open access thrives on information symmetry, openness and transparency.

Contrastly, an intuitive way of exert control in the platform is to assume that producers make socially inefficient decisions and instead decide allocations on their behalf, e.g., Uber as studied in [40]. Dependent on how well the producers understand allocation decisions, [67] showed there may be various outcomes. For example, it is well known and reported in [24] that drivers on ridesharing platforms collaborate and reduce their production to cause demand spikes in the system, often resulting in better individual payoff but worse performance for the platform. It is also common to see drivers on ride-sharing platforms rejecting rides that are requested far from them. This is a risk they are not willing to take as platforms prioritize riders from their point of view is 
studied in [57]. [14, 34, 53, 69] look at other platform means, e.g., through subsidies and incentives, to make sure that drivers participate actively and on only one platform. Under a dynamic twosided facility location model, [6] showed that by imposing a distance constraint, an approximation algorithm can align the interests of the producers and the platform while matching producers and consumers efficiently. Similarly, [12] showed an approximation algorithm to find the optimal allocation and prices to a variety of objective functions. Recent work by [3] aims to characterize the drivers' incentive compatibility conditions for repositioning decisions, and provide new insights on the interplay between admission control and drivers' strategic repositioning decisions. More recently, [56] also considered setting prices that are smooth both in space and time in a spatiotemporal pricing mechanism.

Another possible way to improve on open access is through discriminatory access, where it is studied in [5, 13, 47] that platforms can restrict access between certain producers and markets in a bid to improve market outcomes. A significant amount of effort has been placed into what is known in the literature, e.g., 21, 22, 26, as algorithmic pricing, which is most well known to be exemplified by Amazon's Buybox as studied in [25], where they highlight one seller for each item to every consumer that is looking for it. Another example of discriminatory access is Airbnb's Superhost program studied in [52], which highlights certain renters through badges and have been shown to vastly improve revenue on the peer-to-peer rental platform.

One of the starting objectives of online marketplaces or platforms as stated in [11] was to reduce search costs - that is, to reduce the amount of effort to acquire information on sellers and the quality of their goods. 28] showed that lower search costs leads to an increase in price sensitivity. However, it has also been shown in [19] that too much information revealed in online platforms can instead result in increasing search costs from the consumers' point of view. [62] showed that reducing search costs can lead to higher prices and profit while [36] showed that a monopolist may impede information acquisition to improve her profits. In a more recent work, 29] studies the tradeoff between guiding consumers to their most desired product while also strengthening seller incentives to offer low prices.

Besides access and allocation control, work in this area has also covered a variety of possible design factors, including pricing in [73, competition in [9], reputation in [54, 63, 72], thickness in [10], and also dynamic models for kidney exchange in [4]. Recent empirical studies in [15, 30] also reveal significant price dispersion in online and spatial marketplaces, causing platforms to differentiate products in order to create distinct consumer markets as in [29]. In particular, these results highlight the need to study platforms in using models of networked competition.

\section{Networked Competition.}

Models of networked competition aim to capture the effects of network constraints on strategic interactions between firms. These models include networked Bertrand competition studied in [8, 23, 38, networked Cournot competition in [1,16, 44, networked Stackelberg competition in [50,74, and other networked bargaining games where agents can trade via bilateral contracts over a network that determines the set of feasible trades, e.g., 2, 31, 60,61. These networked competition models have applications in many areas, e.g., electricity markets in [17] and demand-side management in smart grids a.k.a. demand response in [59].

Our work relies on a model of networked Cournot competition. The vast majority of the literature on networked Cournot competition, e.g., 1, 16, 44, focuses on characterizing and computing Nash equilibria, with a recent work of [7] focusing on a scenario with capacity constraints. In a 
similar spirit to the present paper, [46] provide bounds on the worst case efficiency loss of Cournot games, while [20] shows how the mechanism of market clearing (which is determined via the choice of the system operator's objective function) might affect the efficiency of the platform under network constraints. Our work focuses on platform design through access and allocation control under the networked competition model, and extends some key results from [46] on worst case efficiency loss while presenting new results with regards to controlled allocation platforms without network constraints (which already provide key insights with regards to efficiency loss).

\section{Preliminaries}

Our goal in this paper is to analyze the efficiency of online platform designs with regards to access and allocation control. To study this issue it is crucial to consider a setting with networked markets, where the platform allows diverse producers to have access to markets in which they may otherwise not be able to participate.

We describe the competition in online platforms using the networked Cournot competition model introduced in [1] and [16]. As a generalization of the classical model of Cournot competition, the networked Cournot model captures settings in which firms compete to produce a homogeneous good in multiple markets, where each market is potentially only accessible by a subset of firms. The existence of a platform allows firms to participate in a broader set of markets than they could independently. Firms' decisions are connected across markets through an aggregate production cost while decisions across firms are connected via a single price from each market.

The networked Cournot competition model may not capture some important aspects of platform designs, such as the possibility for platforms to set prices or dynamically match agents. However, endogenous price formation allows us to focus on access and allocation control. In fact, the Cournot model has been prominent in many applications, such as analyzing the efficiency loss in electricity markets, e.g., [42,45].

The core of the model is a network specifying the connections between firms and markets. It is described by a directed bipartite graph $(F, M, \mathcal{E})$. Here, we denote by $F:=\{1, \ldots, n\}$ the set of $n$ firms, $M:=\{1, \ldots, m\}$ the set of $m$ markets, and $\mathcal{E} \subseteq F \times M$ the set of directed edges connecting firms to markets where $(i, j) \in \mathcal{E}$ if and only if firm $i$ has access to market $j$.

In general, the efficiency of such marketplaces depends on the structure of the underlying graph, which restricts the set of markets to which each firm has access. A crucial role that the platform might play in this setting is, therefore, the selection of markets that are made available to each firm, potentially allowing all connections through open access or a careful selection through discriminatory access. Additionally, in a controlled allocation platform, an alternative way for a platform to moderate the system is to direct each firm's production to various markets; that is, given the quantity of production decided by the firms, the platform dictates its allocation to the markets. In what follows, we examine these three classes of platform designs: open access platforms, controlled allocation platforms and discriminatory access platforms.

\subsection{Open Access Platforms}

An open access platform allows all firms to freely access all markets. This corresponds to the complete set of directed edges from firms to markets, i.e., $\mathcal{E}=F \times M$. Examples of platforms exhibiting such open access designs include eBay and Etsy, where every customer is shown every 
retailer that sells the item she desires. These platforms thrive on fairness, transparency, and increased production and competition.

To describe a networked Cournot model, we need to define the producer model, market model, and our main objective, social welfare. We introduce these for open access designs below and then discuss how they change for controlled allocation and discriminatory access designs in the sections that follow.

Producer model: Under the open access platform design, each firm decides its production quantity to each market. We let $q_{i j} \in \mathbb{R}_{+}$denote the quantity produced by firm $i$ in market $j$, and let $q_{i}:=\left(q_{i 1}, \ldots, q_{i m}\right) \in \mathbb{R}_{+}^{m}$ denote the supply profile from firm $i$. We also denote the supply profile over all producers as $q:=\left(q_{1}, \ldots, q_{n}\right)$, and define the set of feasible supply profiles from firm $i$ as:

$$
\mathcal{Q}_{i}(\mathcal{E}):=\left\{x \in \mathbb{R}_{+}^{m} \mid x_{j}=0, \forall(i, j) \notin \mathcal{E}\right\} .
$$

Accordingly, the set of feasible supply profiles from all firms is given by $\mathcal{Q}(\mathcal{E}):=\prod_{i=1}^{n} \mathcal{Q}_{i}(\mathcal{E})$. We let $s_{i}$ be the aggregate production quantity of firm $i \in F$. It is given by

$$
s_{i}:=\sum_{j=1}^{m} q_{i j} .
$$

The resulting production cost of firm $i$ is defined by $C_{i}\left(s_{i}\right)$. We assume that the cost function $C_{i}$ is convex, differentiable on $(0, \infty)$, and satisfies $C_{i}(x)=0$ for all $x \leq 0$. Finally, we define $C:=\left(C_{1}, \ldots, C_{n}\right)$ as the cost function profile.

Market model: As is standard in Cournot models of competition, we model price formation according to an inverse demand function in each market. Similar to [16], we restrict our attention to affine inverse demand functions throughout this paper. Specifically, the price in each market $j \in M$ is determined according to $p_{j}\left(d_{j}\right):=\alpha_{j}-\beta_{j} d_{j}$, where $d_{j}$ denotes the aggregate quantity supplied to market $j$, given by

$$
d_{j}:=\sum_{i=1}^{n} q_{i j} .
$$

Here, $\alpha_{j}>0$ measures consumers' maximum willingness to pay, and $\beta_{j}>0$ measures the price elasticity of demand. We describe the price formation here and describe the competition model and equilibrium analysis in Section 2.4.

Social welfare: We measure the performance or efficiency of a platform by the social welfare. For platforms, the pursuit of social welfare benefits both buyers and sellers, and in the long run, promotes the platforms' expansion. For example, Amazon, in its Buy Box design, believes that welfare measures such as availability, fulfillment, and customer service ultimately lead to increased customer satisfaction, and thereby, promote its growth in the long run, as observed in [26].

We adopt the standard notion of social welfare defined as aggregate consumer utility minus the total production cost. Specifically, the social welfare associated with a supply profile $q$ and a cost function profile $C$ is defined as

$$
\operatorname{SW}(q, C):=\sum_{j=1}^{m} \int_{0}^{d_{j}} p_{j}(z) \mathrm{d} z-\sum_{i=1}^{n} C_{i}\left(s_{i}\right),
$$

where $s_{i}$ and $d_{j}$ are defined in Eqs. (1) and (2), respectively. 
We define the optimal social welfare associated with the cost function profile $C$ and the edge set $F \times M$ as:

$$
\mathrm{SW}^{*}(F \times M, C):=\sup _{q \in \mathcal{Q}(F \times M)} \mathrm{SW}(q, C) .
$$

It is straightforward to check that the above supremum can be attained, and that the set of efficient supply profiles is non-empty. One can show that the optimal supply profile can be attained if a social welfare maximizing platform controls both production of firms and their allocation to the different markets, while losing control of either may lead to inefficiency. This definition will be generalized later in the description of discriminatory access platforms.

\subsection{Controlled Allocation Platforms}

The controlled allocation platform design is an attempt to pursue the socially optimal supply profile by controlling one of the two required factors, namely the allocations from firms to markets. In an idealized world, if the firms produce at optimal levels, then the optimal social welfare can be attained. However, in controlled allocation platforms, firms continue to retain control over their production level. For example, on Uber, drivers choose how many hours to work, but largely not the riders they serve.

More formally, a controlled allocation platform is again specified by production cost functions $C_{1}, \cdots, C_{n}$ and inverse demand functions $p_{1}, \ldots, p_{m}$. Each firm chooses its aggregate production quantity $s_{i}$, incurring production cost $C_{i}\left(s_{i}\right)$. The platform then allocates aggregate productions across markets to maximize social welfare, as defined in Eq. 3. a simple argument shows that, at the optimal allocation, all markets that receive a positive supply of production must have the same price. To see this, we analyze the platform's allocation that aims to maximize social welfare. The platform takes the aggregate production $Q=\sum_{i} s_{i}$ (a quantity it has no control over), and performs the following optimization to decide the allocation $d_{j}$ to market $j$, in order to maximize the social welfare:

$$
\begin{aligned}
\max _{d_{1}, d_{2}, \ldots, d_{m}} & \sum_{j=1}^{m} \int_{0}^{d_{j}} p_{j}(z) \mathrm{d} z-\sum_{i=1}^{n} C_{i}\left(s_{i}\right) \\
\text { s.t. } & \sum_{j=1}^{m} d_{j} \leq Q
\end{aligned}
$$

Having no control over the amount of production from the firms, the supplies are fixed, and the optimization is equivalent to maximizing just the consumer surplus, which is the first term in Eq. 3 the definition of social welfare.

By observing the platform's objective, e.g., maximizing social welfare, the firms fully understand how their aggregate productions are to be allocated across the markets, and act together as joint leaders in a Stackelberg or leader-follower game. The formal definition of the Stackelberg game will be presented in Section 2.4. To illustrate such an allocation process, the social welfare maximizing controlled allocation platform allocates starting from the market with the highest willingness to pay and, when that market's price becomes low enough, transitioning into markets with smaller willingness to pay, keeping prices constant across the set of markets receiving positive supplies. In general, an allocation function $A: \mathbf{R}^{+} \rightarrow \mathbf{R}^{m}$ takes as input the aggregate production $Q=\sum_{i \in F} s_{i}$ and outputs the corresponding allocations to the markets $d=\left\{d_{1}, d_{2}, \ldots, d_{m}\right\}$. 
For objectives other than the social welfare, prices may not be the same among markets that receive supplies. We will assume that the platform still pays the same per-unit price $p$ to all firms that produce, where $p$ is the average price over the demand fulfilled, or more formally defined by:

$$
p\left(\sum_{j} d_{j}\right)=\frac{\sum_{j} d_{j}\left(\alpha_{j}-\beta_{j} d_{j}\right)}{\sum_{j} d_{j}} .
$$

\subsection{Discriminatory access platforms}

In contrast to open access platform designs, a discriminatory access platform restricts the set of markets that are accessible by each firm, selecting an edge set $\mathcal{E} \subseteq F \times M$. This restriction may be designed to improve producer surplus, consumer surplus, or total social welfare of the system. An example of a discriminatory access platform design is Amazon's Buy Box, where Amazon chooses a default seller based on a score that combines pricing, availability, fulfillment, and customer service. This prevents certain firms from accessing certain markets. In a dicriminatory access platform, each firm $i$ can freely decide the quantity $q_{i j}$ it supplies to each market $j$, with the restriction that $q_{i j}=0$ for all $(i, j) \notin \mathcal{E}$. We define the set of feasible supply profiles from firm $i$ as:

$$
\mathcal{Q}_{i}(\mathcal{E}):=\left\{x \in \mathbb{R}_{+}^{m} \mid x_{j}=0, \forall(i, j) \notin \mathcal{E}\right\} .
$$

Accordingly, the set of feasible supply profiles from all firms is given by $\mathcal{Q}(\mathcal{E}):=\prod_{i=1}^{n} \mathcal{Q}_{i}(\mathcal{E})$.

Under the discriminatory access platform design, the supplier cost functions and market price formation follows from the open access platform design, and its competition model is described in Section 2.4. Refining on the open access platform design case, the efficient social welfare associated with an edge set $\mathcal{E}$ and a cost function profile $C$ is

$$
\mathrm{SW}^{*}(\mathcal{E}, C):=\sup _{q \in \mathcal{Q}(\mathcal{E})} \operatorname{SW}(q, C) .
$$

A supply profile $q \in \mathcal{Q}(\mathcal{E})$ is said to be efficient with respect to a fixed edge set $\mathcal{E}$, or $\mathcal{E}$-efficient, if it satisfies $\mathrm{SW}(q, C)=\mathrm{SW}^{*}(\mathcal{E}, C)$. Again, it is straightforward to check that the above supremum can be attained, and that the set of efficient supply profiles is non-empty.

\subsection{Networked Competition Model}

We now describe the competition models and their equilibria for each of the three designs.

\subsubsection{Competition under open and discriminatory access platforms:}

We consider profit maximizing firms, where the profit $\pi_{i}$ of a firm $i$, given the supply profiles of all other firms $q_{-i}=\left(q_{1}, . ., q_{i-1}, q_{i+1}, . ., q_{n}\right)$, is given by

$$
\pi_{i}\left(q_{i}, q_{-i}\right):=\sum_{j=1}^{m} q_{i j} p_{j}\left(d_{j}\right)-C_{i}\left(s_{i}\right),
$$

where $p_{j}$ is a price formed based on the total demand at node $j$, coupling the decisions made between different producers. We denote by $\pi:=\left(\pi_{1}, \ldots, \pi_{n}\right)$ the collection of payoff functions of 
all firms. The triple $(F, \mathcal{Q}(\mathcal{E}), \pi)$ defines a normal-form game applicable for the open access and discriminatory access designs, which we refer to as the networked Cournot game associated with the edge set $\mathcal{E}$. We use Nash equilibrium to describe the stable outcome of the networked Cournot game. It is defined as follows.

Definition 2.1. A supply profile $q \in \mathcal{Q}(\mathcal{E})$ constitutes a pure strategy Nash equilibrium of the game $(F, \mathcal{Q}(\mathcal{E}), \pi)$ if for every firm $i \in F, \pi_{i}\left(q_{i}, q_{-i}\right) \geq \pi_{i}\left(\bar{q}_{i}, q_{-i}\right)$, for all $\bar{q}_{i} \in \mathcal{Q}_{i}(\mathcal{E})$.

Under the assumptions of convex cost functions and affine inverse demand functions, [1] has shown that the networked Cournot game is an ordinal potential game. Additionally, it admits a unique Nash equilibrium that is the unique optimal solution to a convex program. We summarize the results of [1] in the following lemma.

Lemma 2.1. [1] The game $(F, \mathcal{Q}(\mathcal{E}), \pi)$ admits a unique Nash equilibrium $q^{N E}(\mathcal{E})$ that is the unique optimal solution to the following convex program:

$$
\underset{q \in \mathcal{Q}(\mathcal{E})}{\operatorname{maximize}} \operatorname{SW}(q, C)-\sum_{i=1}^{n} \sum_{j=1}^{m} \frac{\beta_{j} q_{i j}^{2}}{2} .
$$

In general, the supply profile at the unique Nash equilibrium differs from the efficient supply profile. Such an efficiency loss is usually measured according to the price of anarchy of the game that was first introduced in [48]. In the rest of this article, we use the terminologies worst case efficiency loss and price of anarchy interchangeably.

Definition 2.2. The price of anarchy associated with the edge set $\mathcal{E}$, the cost function profile $C$, and the corresponding networked Cournot game $(F, \mathcal{Q}(\mathcal{E}), \pi)$ is defined as

$$
\rho(\mathcal{E}, C):=\frac{\mathrm{SW}^{*}(F \times M, C)}{\operatorname{SW}\left(q^{\mathrm{NE}}(\mathcal{E}), C\right)} .
$$

where $F \times M$ refers to the complete bipartite graph and we set $\rho(F \times M, C)=1$ if $\mathrm{SW}^{*}(\mathcal{E}, C)=0$ and $\mathrm{SW}\left(q^{\mathrm{NE}}(\mathcal{E}), C\right)=0$. Note here that comparisons are always made against the optimal social welfare, i.e., that attained with the complete bipartite graph $F \times M$.

Since the Nash is unique for the networked Cournot game, we do not explicitly define price of anarchy or the worst case efficiency loss over all possible equilibrium. In general, for games with possible multiplicity of Nash equilibria, the price of anarchy is defined as the ratio of the efficient social welfare over that of the Nash equilibrium with the lowest social welfare, and is no less than 1.

\subsubsection{Competition under controlled allocation platforms:}

Competition in controlled allocation platforms is, instead, described according to a Stackelberg competition model. A Stackelberg competition model includes a leader, who moves first, and a follower, who moves sequentially after. The leader is assumed to know the best response of the follower (dependent on the move it makes), and solves by backward induction the optimal choice to make, i.e., the quantity to produce. In the networked Stackelberg game, the firms act jointly as leaders deciding their production, and the platform best responds by allocating the aggregate production to maximize an objective made known beforehand to the firms, e.g., social welfare. 
Having defined the allocation function $A$ and the price $p$ in Eq. [5, we can now define the profit $\pi_{i}$ of a firm $i$ under the platform allocation $A$ as

$$
\pi_{i}\left(s_{i}, s_{-i}, A\right)=s_{i} p\left(A\left(\sum_{i} s_{i}\right)\right)-C_{i}\left(s_{i}\right)
$$

Definition 2.3. Given the platform allocation function A, a supply profile $s^{S E}$ constitutes a Stackelberg equilibrium of the networked Stackelberg game if for every firm $i, \pi_{i}\left(s_{i}^{S E}, s_{-i}^{S E}\right) \geq$ $\pi_{i}\left(s_{i}, s_{-i}^{S E}\right), \forall s_{i} \geq 0$.

Unlike the networked Cournot competition for the access control designs, Lemma 2.1 above does not hold since the networked Stackelberg game for the controlled allocation design is significantly different in that firms do not control their allocations and that there can be multiple equilibria instead. Additionally, the worst case efficiency loss or price of anarchy in that case is defined similarly, but is taken as the maximum multiplicative factor $\rho$ over all Stackelberg equilibria.

Definition 2.4. The price of anarchy $\rho(C, M)$ associated with the networked Stackelberg game is defined as

$$
\rho(C, M)=\frac{\mathrm{SW}^{*}(F \times M, C)}{\mathrm{SW}\left(s^{N E}\right)},
$$

for the worst Stackelberg equilibrium (when it exists) $s^{S E}$ under a set of cost functions $C$ and a set of markets $M$.

The controlled allocation setting is similar to a networked Stackelberg competition introduced in [20,74] whereby the platform first commits to allocations based on its objective and the firms act first by selecting their production, privy to how the platform distributes production. Under the networked Stackelberg competition, there exists systems with (i) no Stackelberg equilibrium, (ii) a unique Stackelberg equilibrium, and (iii) multiple Stackelberg equilibria.

\section{Open Access Platforms}

Open access platforms such as eBay and Etsy thrive on transparency and offer buyers/consumers access to all possible sellers. This platform design is usually accompanied by lower entry costs that typically improve competition between producers, and thereby boost transaction volume, and oftentimes lead to socially efficient markets by fairly presenting similar opportunities to every participant.

In this section, we aim to provide tight bounds on the loss of welfare due to selfish behavior in networked Cournot games under open access platform designs. The main theorem in this section provides a (tight) efficiency loss bound of a networked Cournot game governed by an open access platform with respect to the number of firms $n$.

Theorem 3.1. The worst case efficiency loss associated with a cost function profile $C$ and the corresponding open access networked Cournot game $(F, \mathcal{Q}(F \times M), \pi)$ is upper bounded by

$$
\rho(F \times M, C) \leq \frac{3}{2}\left(1-\frac{1}{3 n+6}\right) .
$$


The bound is tight if markets have the same maximal willingness to pay, i.e., $\alpha_{1}=\alpha_{2}=\cdots=\alpha_{m}$. Precisely, for any choice of $n$, there exists a cost function profile $\bar{C}$, such that

$$
\rho(\bar{C})=\frac{3}{2}\left(1-\frac{1}{3 n+6}\right) .
$$

This result generalizes those in [46] from a single market case to the networked setting, succinctly presented in the following corollary to Theorem 3.1 .

Corollary 3.1.1. Open access platforms have worst case efficiency loss of at most 3/2.

In the remainder of this section, we prove Theorem 3.1 first by proving a critical result in Lemma 3.2 that the cost functions maximizing the worst case efficiency loss are linear ones, a result presented in [46] for the case without a network. That means that given any set of convex cost functions, we can find a corresponding set of linear cost functions that increase the worst case efficiency loss. Applying Lemma 3.2, bounds on efficiency loss are developed for both the symmetric and asymmetric costs setting in Proposition 3.1 (Section 3.2.1) and Theorem 3.1 (Section 3.2.2) respectively.

The result in Theorem 3.1 (for firms with asymmetric costs) is potentially counter-intuitive since an increase in the number of firms $n$ (presumably increasing competition) results instead in a larger worst case efficiency loss. To shed more light on this, we further develop worst case efficiency loss bounds on networked Cournot games under open access platforms with linear cost functions and bounds on asymmetry, presented later in Proposition 3.2. Additionally, in the symmetric cost case, in Proposition 3.1 we see that it is indeed that as the number of perfectly competing firms increase, the worst case efficiency loss decreases.

We present a generalized result from [64] in the following lemma that demand fulfilled at the Nash equilibrium of the networked Cournot game for any market is at least half of the fulfilled demand under a socially optimal allocation.

Lemma 3.1. For any market $j \in M$, the demand fulfilled at Nash is at least half of the demand fulfilled at the social optimal allocation.

The lemma underlines one of the critical reasons open access platforms perform well — that open access platform designs preserve a large proportion of production. However, open access platforms still suffer from selfish behavior that manifest in the misalignment between optimal allocations and Nash allocations. Contrasting to this, in the next section, we explore a platform targeted at perfect allocation.

\subsection{Identifying the Worst-case Cost Function Profile}

The following lemma reveals that the worst case efficiency loss of networked Cournot games are maximized at a cost function profile consisting of cost functions that are linear over the nonnegative real numbers, demonstrating that any cost function profile $C$ as specified in Section 2.1 can be correspondingly re-designed to construct a piecewise linear cost function profile $\bar{C}$, resulting in an increased worst case efficiency loss. In light of this result, in designing efficiency loss bounds guaranteed to hold for all cost functions belonging to the family specified in Section 2.1, it suffices to consider cost functions that are linear on $(0, \infty)$. 
Lemma 3.2. Given a cost function profile $C$, define the cost function profile $\bar{C}=\left(\bar{C}_{1}, \ldots, \bar{C}_{n}\right)$ according to

$$
\bar{C}_{i}\left(s_{i}\right)=\left(\partial^{+} C_{i}\left(\sum_{j=1}^{m} q_{i j}^{\mathrm{NE}}(F \times M)\right) \cdot s_{i}\right)^{+}
$$

for $i=1, \ldots, n$, where $\partial^{+} C_{i}$ denotes the right-derivative of the function $C_{i}$. It holds that $\rho(F \times$ $M, C) \leq \rho(F \times M, \bar{C})$.

We provide some intuition here behind the proof of Lemma 3.2, while the full proof can be found in the Appendix. The social welfare achieved at a Nash equilibrium depends on the underlying cost function profile only at the marginal cost at Nash equilibrium, which follows from the fact that each firm's profit is a concave function of her own supply profile. By replacing the original convex cost functions by linear cost functions which have the same marginal cost at Nash equilibrium, the resulting Nash equilibrium has identical production quantities and a worse-off social welfare compared to the original one. A careful reader may notice here that the efficient social welfare may also decrease, but one can show that the decrease in the efficient social welfare is is guaranteed to be larger than that of the social welfare at Nash equilibrium.

\subsection{Efficiency Loss in Open Access Platforms}

The characterization of the worst-case or optimal cost function profile in Lemma 3.2 is crucial to the derivation of tight upper bounds on the worst case efficiency loss bounds for networked Cournot games. One example of this simplification is that under linear cost function profiles, the networked Cournot game can essentially be decoupled into $m$ many different Cournot games with a single market since the marginal cost of each firm remain constant. In what follows, we examine the role played by (a)symmetry in linear cost functions in determining platform efficiency.

Before proving Theorem 3.1, we prove a result for the case where firms have symmetric costs. This help us show that the intuition that increased competition does indeed lead to less efficiency loss is indeed correct given the right assumptions.

In proving Theorem 3.1, we devise worst case asymmetric cost functions compared to the one cost function utilized in the optimal case. Intuitively, since the optimal would only be utilizing the firm with the smallest marginal cost, the worst case family of cost functions should consist of one firm with a low cost and the remaining with high costs. As we increase the number of firms in this scenario, the set with high costs increase, leading to a slight increase in competition which however is inefficient. Another way to approach the counter-intuitive result is that the additional firm can always be designed to have arbitrarily large marginal cost, such that it does not participate, and therefore maintains the efficiency loss without its participation.

The worst case efficiency loss bounds under these two families of cost functions exhibit distinct behaviors in terms of their monotonicity in the number of firms $n$. We thus further explore the question as to how the degree of asymmetry between firms' cost functions might affect the efficiency loss in open access platforms. In doing so, we characterize tight worst case efficiency loss bounds when the underlying cost functions are restricted to be linear over $(0, \infty)$, and whose slope lies within a bounded interval $\left[c_{\min }, c_{\max }\right] \subseteq \mathbb{R}_{+}$. 


\subsubsection{Symmetric Cost Functions}

Our analysis begins with the setting where firms have identical cost functions, representing equally competitive producers. We establish a tight upper bound on the worst case efficiency loss under this setting in Proposition 3.1 that monotonically decreases in the number of firms, and converges to 1 as the number of producers grows large. This conforms with the intuition that increasing (symmetric/perfectly competitive) suppliers strictly increases competition, and thereby reduce the extent to which any one producer might exert market power, improving the efficiency of platforms as selfish behavior becomes restricted.

Proposition 3.1. For firms with symmetric costs, i.e., $C_{i}=C_{j}, \forall i, j \in F$, then the efficiency loss associated with the corresponding open access networked Cournot game $(F, \mathcal{Q}(F \times M), \pi)$ is bounded above by

$$
\rho(F \times M, C) \leq 1+\frac{1}{(n+1)^{2}-1} .
$$

In addition, this bound is tight, i.e., for any choice of $n$, there exists a symmetric cost function profile with a corresponding worst case efficiency loss equal to the upper bound.

In proving Proposition 3.1, a direct application of Lemma 3.2 reveals that the worst-case symmetric cost function profile consists of $n$ identical cost functions that are linear on $(0, \infty)$. The details of the proof are deferred to the Appendix.

\subsubsection{Arbitrary Asymmetric Cost Functions}

We now consider the more general setting in which firms have arbitrary asymmetric cost functions satisfying the assumptions in Section 2.1, summarized in Theorem 3.1, where we establish a tight upper bound on the efficiency loss that is monotonically increasing in the number of firms. In this case, it may seem counterintuitive that a perceived increased competition instead lead to efficiency loss. Its proof is provided in the Appendix.

This seemingly counterintuitive result can occur if an expensive firm enters the market. First, note that the entry of this new firm results in an increase in aggregate supply at Nash equilibrium, because of increased 'competition' in the market. However, its entry takes away production from its (cheap) competitors. This manifests in a reduction in social welfare if the increase in production cost exceeds the increase in consumer utility. Such a phenomenon is known as the "excess entry theorem" in the economics literature, e.g., [49, 58, 71], and reveals the possibility that a new firm's entry can lead to a reduction in social welfare.

Additionally, taking the number of firms $n \rightarrow \infty$ yields a worst case efficiency loss bound that is valid for any number of firms, and any number of markets, presented in Corollary 3.1.1. This recovers the $3 / 2$ worst case efficiency loss or price of anarchy bound first established by [46] for a single market. In fact, under this generalized characterization, one can obtain both the previous result in their work, and for a networked market of any size with open access.

\subsubsection{Linear Cost Functions with Bounds on Asymmetry}

The efficiency loss results in Proposition 3.1 and Theorem 3.1 appear contradictory at first sight. Namely, the efficiency loss bound is decreasing in $n$ if producers have symmetric cost functions but 
it is increasing in $n$ if producers are allowed to have asymmetric cost functions. In what follows, we explore how the efficiency loss depends on the asymmetry between firms' cost functions, providing more intuition towards this counter-intuitive result, by restricting ourselves to cost functions that are linear on $(0, \infty)$, and whose slopes lie within $\left[c_{\min }, c_{\max }\right] \subseteq \mathbb{R}_{+}$.

$$
\mathcal{L}\left(c_{\min }, c_{\max }\right):=\left\{C_{0}: \mathbb{R} \rightarrow \mathbb{R}_{+} \mid C_{0}(x)=(c x)^{+}, c \in\left[c_{\min }, c_{\max }\right]\right\} .
$$

We write $C \in \mathcal{L}^{n}\left(c_{\min }, c_{\max }\right)$ if the cost function profile $C$ satisfies $C_{i} \in \mathcal{L}\left(c_{\min }, c_{\max }\right)$ for each firm $i \in F$. It will be convenient to define a non-dimensional parameter $\gamma_{j}$, which measures the degree of (a)symmetry between firms for each market $j \in M$. Specifically, for each market $j \in M$, define

$$
\gamma_{j}:=1-\frac{c_{\max }-c_{\min }}{\alpha_{j}-c_{\min }}
$$

It holds that $\gamma_{j} \in(-\infty, 1]$ if $c_{\min }<\alpha_{j}$. Clearly, $\gamma_{j}$ is increasing in consumers' maximum willingness to pay $\alpha_{j}$, and decreasing in the maximum cost $c_{\text {max }}$. It follows that a value of $\gamma_{j}$ close to one implies a small degree of asymmetry between firms' cost functions relative to consumers' maximum willingness to pay in market $j$.

The following proposition provides a tight bound on the worst case efficiency loss bound when firms have linear cost functions with a bounded degree of asymmetry.

Proposition 3.2. Let $C \in \mathcal{L}^{n}\left(c_{\min }, c_{\max }\right)$, and assume that $c_{\min }<\max _{j \in M} \alpha_{j}$ (that at least one firm would be willing to produce to one market). The worst case efficiency loss associated with the corresponding open access networked Cournot game $(F, \mathcal{Q}(F \times M), \pi)$ is upper bounded by

$$
\rho(F \times M, C) \leq \frac{\sum_{j=1}^{m} \frac{\left(\left(\alpha_{j}-c_{\min }\right)^{+}\right)^{2}}{\beta_{j}}}{\sum_{j=1}^{m}\left(\frac{2 n+4}{3 n+5}+\delta\left(\gamma_{j}, n\right)\right) \frac{\left(\left(\alpha_{j}-c_{\min }\right)^{+}\right)^{2}}{\beta_{j}}},
$$

where the function $\delta(\gamma, n)$ is defined according to

$$
\delta(\gamma, n)= \begin{cases}0 & \text { if } \gamma<\frac{2 n+3}{3 n+5} \\ \frac{(n-1)(3 n+5)}{(n+1)^{2}}\left(\gamma-\frac{2 n+3}{3 n+5}\right)^{2} & \text { otherwise }\end{cases}
$$

The bound is tight if $\alpha_{1}=\alpha_{2}=\cdots=\alpha_{m}$.

The worst case efficiency loss bound specified in Proposition 3.2 depends on the degree of (a)symmetry between firms' cost functions only through the terms $\delta\left(\gamma_{j}, n\right)$ for $j=1, \ldots, m$. In particular, as $\delta(\gamma, n)$ is non-decreasing in $\gamma$, a reduction in the degree of asymmetry between firms' cost functions manifests in a reduction in the worst case efficiency loss bound.

With the positive worst case efficiency loss results from open access platform designs, together with the result in Lemma 3.1 that open access platform designs preserve a good proportion of demand fulfilled, we explore in the next section the platform design that controls allocations on behalf of the participating firms, allowing them to only decide their production quantity. 


\section{Controlled Allocation Platforms}

Despite preserving large production rates, the open access platform design suffers from some efficiency loss through misaligned allocations. We design controlled allocation platforms to aggregate production and perfectly allocate to various markets in hope that this control circumvents the misaligned allocations. Unlike the open access platform described in Section 3, a controlled allocation platform allocates the production to different markets in a socially optimal way for any given aggregate production quantity.

However, controlled allocation platforms are not as simple to optimize as they seem. In particular, the control over allocations exerted by the platform may create unintended incentives incentives for firms to withhold production, as empirically shown in 24 for the ride-sharing platform Uber. Though firms cannot strategically choose prices or matches, they retain control over their participation in the platform. In the networked Stackelberg setting, this takes the form of strategic choices of production levels, with the platform only allocating on their behalf to maximize a certain quantity, e.g., social welfare.

Our main result in this section highlights that distorted incentives in controlled allocation platforms can lead to inefficient market outcomes that can be far worse than outcomes under open access designs.

Theorem 4.1. A controlled allocation platform maximizing social welfare can have unbounded price of anarchy. In particular, there exists a family of networks where the Stackelberg equilibrium is unique and the worst case efficiency loss is $\Omega(m)$, i.e., there exists $s^{S E}$ and cost functions $C$ such that:

$$
\rho(C, M) \geq \Omega(m)
$$

The contrast between Theorem 4.1 for controlled allocation platforms and Theorem 3.1 for open access platforms is stark. While open access platforms preserves at least $2 / 3$ of the optimal social welfare regardless of market parameters, controlling allocations can lead to reactive producer behavior which drives efficiency preservation rates possibly all the way to 0 . In this section, we first prove Theorem 4.1. Additionally, we provide a generalization of Theorem 4.1 in Theorem 4.2 , which shows that controlled allocation platforms remain inefficient even if the platform chooses different objective functions in clearing the market.

\subsection{Social Welfare Maximizing Controlled Allocation Platforms}

Given the results from Section 3, one may intuitively think that perfect allocation can lead to improved efficiency loss bounds. In Theorem 4.1, we show that such a platform, even with the best intentions, i.e., maximizing social welfare, can lead instead to an unbounded efficiency loss. On the other hand, allowing for an open access design and not controlling allocations lead to a bounded worst case efficiency loss as in Section 3 .

Note that the networks constructed to prove Theorem 4.1 are simple. They use a single firm with costless production. The construction begins with a single market and then, as markets are added one by one to the system, the parameters of each new market are such that the firm has no incentive to increase production due to the reallocation under the controlled allocation, whereas the socially optimal (non-Nash) production level does increase, as does the optimal welfare. Note that, when a firm has no production cost, the socially optimal production is always to produce until the price in every market is driven to 0. 
Theorem 4.1 is proven using a construction which highlights that an efficient platform should prevent firms from withholding production, which may be achieved by modifying the market clearing mechanism appropriately. This begs the question: Can the platform do better by optimizing a different quantity? We now show that the efficiency loss in controlled allocation platforms remain unbounded when the platform chooses a family of alternative objective functions in clearing the market. In particular, such a family of objective functions are given by convex combinations of social welfare and consumer surplus.

\subsection{Consumer Surplus Maximizing Controlled Allocation Platforms}

Before attending to the family of objectives, we first consider the corner case in consumer surplus, which helps give some intuition as to why the worst case efficiency loss can grow large. Given a fixed supply, the consumer surplus is defined as follows.

Definition 4.1. Specifically, the consumer surplus associated with a supply profile $q$ is defined according to

$$
C S(q):=\sum_{j=1}^{m} \int_{0}^{d_{j}} p_{j}(z) d z-d_{j} p_{j}\left(d_{j}\right),
$$

where $d_{j}$ is defined in Eq. (2).

In fact, the worst case efficiency loss is unbounded under the objective of consumer surplus.

Proposition 4.1. A controlled allocation platform maximizing consumer surplus have infinitely large price of anarchy. In particular, even under a two market, one firm setting, the price of anarchy is $\infty$.

The idea behind the two node construction which proves the proposition is simple: Maximizing consumer surplus implies that the platform is agnostic to the maximal willingness to pay, whereas rational producers consider that. A simple example of this is a one firm, two market network has the firm with linear cost $c s$, and the markets have parameters

$$
\alpha_{1}=c+\epsilon, \beta_{1}=\epsilon / 2, \alpha_{2}=c-\epsilon, \beta_{2}=\epsilon,
$$

which results in the platform allocating fully to the second market before accessing the first market. It is trivial to show that being forced to participate in the whole of the second market, the firm would rather not produce anything at all. On the other hand, the optimal allocation has 2 units in the first market and none in the second, resulting in a positive social welfare, and therefore an unbounded worst case efficiency loss.

\subsection{A General result on Controlled Allocation Platforms.}

The control over allocations is delicate, and explicitly not optimizing (as in the open access case) is more efficient than optimizing either social welfare or consumer surplus. To understand how the worst case efficiency loss is affected by different objective functions, we now extend the analysis of 
the social welfare case to consider an objective $\operatorname{OBJ}(\lambda)$ to be a convex combination of consumer surplus and revenue, parametrized by $\lambda$ :

$$
\operatorname{OBJ}(\lambda)=\sum_{j \in M}\left[\lambda\left(\int_{0}^{d_{j}} p_{j}(q) d q-d_{j} p_{j}\left(d_{j}\right)\right)+(1-\lambda)\left(d_{j} p_{j}\left(d_{j}\right)\right)\right]
$$

For example, when $\lambda=1$, the above objective is consumer surplus whereas $\lambda=0$ gives revenue, and when $\lambda=0.5$, the resulting objective is half of consumer welfare, which is equivalent to a social welfare objective. In particular, we are able to provide a lower bound on the worst case efficiency loss for the controlled allocation platform maximizing the convex combination of objective functions.

We present a more general version of the previous results on consumer surplus $(\lambda=1)$ and social welfare $(\lambda=1 / 2)$, where worst case efficiency loss is infinitely large and growing linearly in the number of markets respectively. This result is presented in the following theorem.

Theorem 4.2. The worst case efficiency loss $\rho(k)$ of controlled allocation platforms optimizing various objective functions (characterized by $\sigma=\frac{2-3 \lambda}{1-\lambda}$ ) satisfies:

$$
\rho(\sigma) \geq\left\{\begin{array}{l}
\infty, \text { if } \sigma \leq 0 \\
\Omega(m), \text { if } 0<\sigma \leq 1 \\
\max \left\{\frac{2}{3 \sqrt{\frac{1}{\sigma}\left(1-\frac{1}{\sigma}\right)}}, \frac{3}{2}\right\}, \text { otherwise. }
\end{array}\right.
$$

This means that the construction developed to prove Theorem 4.1 also can be used to show that any objective between consumer surplus and social welfare leads to similar results to the social welfare case in Theorem 4.1 and that past social welfare, the upper bound for worst case efficiency loss decreases towards a nice constant, which corresponds to the open access result.

When considering linear inverse demand functions and assuming $d_{j} \leq \alpha_{j} / \beta_{j}$, the above objective can be simplified to the following after dividing by a constant $(1-\lambda)$ assuming $\lambda \neq 1$ (having dealt with the consumer surplus case in the previous subsection),

$$
\operatorname{OBJ}(\lambda)=\sum_{j \in M}\left[\frac{\lambda}{2(1-\lambda)} \beta_{j} d_{j}^{2}+\left(\alpha_{j} d_{j}-\beta_{j} d_{j}^{2}\right)\right]
$$

Observing the stationarity conditions with respect to $d_{j}$, we find that the platform maximizing such an objective would keep the following quantity constant across markets:

$$
\frac{\delta \mathrm{OBJ}(\lambda)}{\delta d_{j}}=\alpha_{j}-\frac{2-3 \lambda}{1-\lambda} \beta_{j} d_{j}
$$

which confirms that as $\lambda$ approaches 1 , the emphasis on the maximal willingness to pay $\alpha_{j}$ decreases, and becomes potentially inefficient. Increasing past the point $\lambda=2 / 3$, the optimization function also changes from a concave maximization problem to a convex one, i.e., the problem ends up being a (nonconvex) Quadratic Program (QP). This also reveals that whenever a new market is accessed (regardless of $\lambda<1$ ), that the following relationship is preserved for any new additional supply $q_{j}, q_{k}, \beta_{j} q_{j}=\beta_{k} q_{k}$, with the parameter $\lambda$ basically dictating when the next market is accessed. For example, when $\lambda=0.5$, prices are kept constant for each participated market.

The issue with controlled allocation platforms is that the platform's market clearing mechanism is agnostic to underlying cost functions. While this action seemingly makes much sense under this 
setting, we show above that having the wrong objectives can yield (in the extreme) unbounded or infinitely large price of anarchy, preserving close to or none of the optimal social welfare. It should be clear through the section that such a strategy of controlling allocations potentially leads to undesirable and reactive producer behavior. As such, an alternative market clearing mechanism is necessary.

\section{Discriminatory Access Platforms}

Open access designs preserve large productions but suffer from misaligned allocations, while controlled allocation designs lead to undesirable and unintended incentives for the producers to curtail production. Recently, some platforms have started shifting from open access platform designs towards more delicate designs which aim to both retain the incentive to produce in open access platforms and exert some control over allocations. Results from this section show the promise of some of these changes. We design such a platform to be similar to mechanisms such as Amazon's Buybox and Airbnb's Superhost, which highlights particular sellers or renters while hiding others.

In the setting of our model, such access control corresponds to the specification of the edge set of the bipartite graph that connect firms to markets, with the goal of maximizing social welfare at the unique Nash equilibrium of the resulting networked Cournot game. This platform is referred to as a discriminatory access platform in this paper. Unlike the controlled allocation platform, the efficiency of discriminatory access platforms is guaranteed to be no worse than open access platforms, as open access is a valid choice of access control.

Our work on discriminatory access design culminates in the following theorem, highlighting the bounded efficiency loss from discriminatory access platforms at the optimal network design. We prove a complementary result in Theorem 5.2 that this optimal network design problem can be computed for the worst case cost functions via a greedy algorithm presented later in the section.

Theorem 5.1. Assume that each firm's cost function is linear over $(0, \infty)$, and assume that $\mathcal{E}^{*}$ is an the optimal network design for the discriminatory access platform. Let $C \in \mathcal{L}^{n}\left(c_{\min }, c_{\max }\right)$, and assume that $c_{\min }<\max _{j \in M} \alpha_{j}$. The efficient social welfare associated with the edge set $\mathcal{E}^{*}$ satisfies

$$
\mathrm{SW}^{*}\left(\mathcal{E}^{*}, C\right)=\mathrm{SW}^{*}(F \times M, C) .
$$

The efficient social welfare can be attained at the edge set $\mathcal{E}^{*}$. Moreover, the worst case efficiency loss associated with the discriminatory access networked Cournot game $\left(F, \mathcal{Q}\left(\mathcal{E}^{*}\right), \pi\right)$ is upper bounded by

$$
\rho\left(\mathcal{E}^{*}, C\right) \leq \frac{\sum_{j=1}^{m} \frac{\left(\left(\alpha_{j}-c_{\min }\right)^{+}\right)^{2}}{\beta_{j}}}{\sum_{j=1}^{m} \max _{k \in\{1, . ., n\}}\left\{\frac{2 k+4}{3 k+5}+\delta\left(\gamma_{j}, k\right)\right\} \frac{\left(\left(\alpha_{j}-c_{\min }\right)^{+}\right)^{2}}{\beta_{j}}},
$$

where the function $\delta(\gamma, n)$ is defined in Proposition 3.2, and serves as a measure of the degree of asymmetry between firms' cost functions.

The above bound is tight if $\alpha_{1}=\alpha_{2}=\cdots=\alpha_{m}$.

The functions that maximize worst case efficiency loss in networked Cournot games are linear cost functions. Therefore, by optimizing based on the linear cost functions, we are sure the bound in Theorem 5.1 also applies to cases where firms have general convex costs. Additionally, choosing the 
number of firms $n=1$ yields a worst case efficiency loss bound of $4 / 3$ for (optimized) discriminatory access platforms with any number of firms and markets, improving upon the $3 / 2$ price of anarchy bound for open access platforms established in Corollary 3.1.1. The result is formally stated as follows.

Corollary 5.1.1. Assume that each firm's cost function is linear over $(0, \infty)$. Discriminatory access platforms have worst case efficiency loss of at most $4 / 3$.

In general, we show that choosing an optimal edge set that maximizes social welfare at Nash equilibrium amounts to a mathematical program with equilibrium constraints (MPEC), and is, in general, computationally intractable.

\subsection{The Optimal Network Design Problem}

The optimal network design problem amounts to the selection of an edge set $\mathcal{E}$, which maximizes social welfare at the unique Nash equilibrium of the resulting networked Cournot game. Formally, Lemma 2.1 provides a characterization of the supply profile at the unique Nash equilibrium of the game $(F, \mathcal{Q}(\mathcal{E}), \pi)$ as the unique optimal solution to a convex program. Therefore, the optimal network design problem admits a formulation as the following MPEC:

$$
\begin{aligned}
& \text { maximize } \operatorname{SW}(q, C) \\
& \text { subject to } \quad \mathcal{E} \subseteq F \times M \\
& q \in \underset{x \in \mathcal{Q}(\mathcal{E})}{\arg \max }\left\{\operatorname{SW}(x, C)-\sum_{j=1}^{m} \sum_{i=1}^{n} \frac{\beta_{j} x_{i j}^{2}}{2}\right\}
\end{aligned}
$$

Here, the decision variables are the edge set $\mathcal{E}$ and the supply profile $q$. The challenge in solving the above problem stems from the equilibrium constraint on $q$, and the presence of the discrete decision variable $\mathcal{E}$. (An equilibrium constraint requires that a vector be an optimal solution to a optimization problem. In general, this leads to a nonconvex and disconnected feasible region for MPECs. See [55] for a more detailed discussion.) In what follows, we show that, considering linear cost functions which represents the worst case efficiency loss, the above problem can be solved using a greedy algorithm.

\subsection{Greedy Algorithm for Optimal Worst-case Network Design}

In this section, we restrict ourselves to cost functions that are linear on $(0, \infty)$, i.e., the ones maximizing worst case efficiency loss. Specifically, we assume that the cost function of each firm $i \in F$ satisfies $C_{i}\left(s_{i}\right)=\left(c_{i} s_{i}\right)^{+}$, where $c_{i} \geq 0$. Leveraging on this assumption, we propose a greedy algorithm for solving the optimal network design problem (9) in Algorithm 1. For each market $j \in M$, the greedy algorithm visits firms in ascending order of marginal cost, and provides each firm it visits access to market $j$ if its inclusion in that market increases social welfare.

Clearly, Algorithm 1 yields an edge set $\mathcal{E}^{*}$, whose corresponding Nash equilibrium has a social welfare that is no smaller than that of the open access platform.

Theorem 5.2. If $\mathcal{E}^{*}$ is an edge set obtained from Algorithm 1 , then $\left(\mathcal{E}^{*}, q^{N E}\left(\mathcal{E}^{*}\right)\right)$ is an optimal solution to (11), that is, the greedy algorithm achieves the optimal network design. 


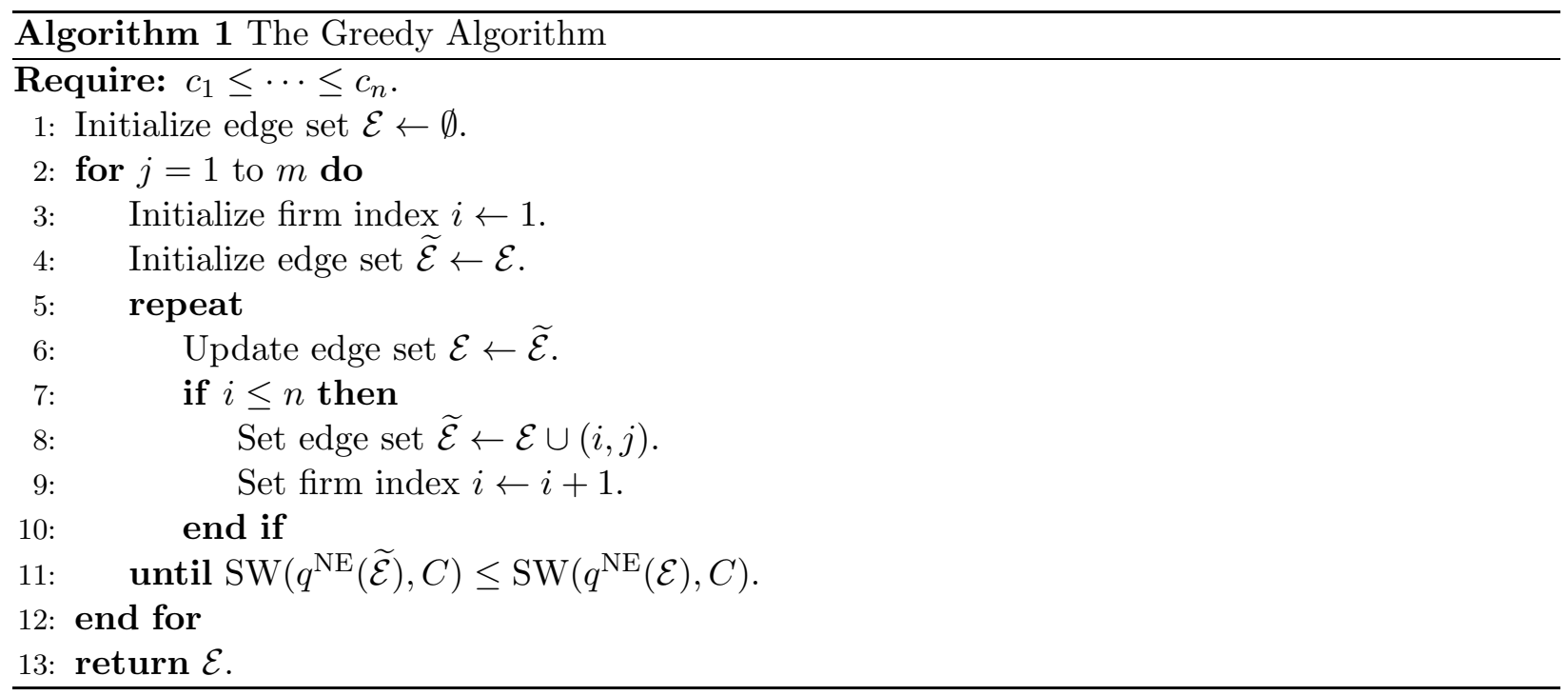

Theorem 5.1 reveals the advantage discriminatory access platforms have over open access ones in reducing the efficiency loss at Nash equilibrium, while Theorem 5.2 guarantees we can find such an edge set when optimizing over the worst case network design. Namely, when the edge set is chosen to be an optimal solution of the network design problem (9), the discriminatory access platform is guaranteed to have a tight bound on the price of anarchy that is no larger than that of the open access platform. Moreover, this price of anarchy bound is guaranteed to be non-increasing in the number of firms $n$.

We observe under our model that sacrificing transparency and discriminating choices of firms for different markets allow for a slight improvement in the worst case social welfare preserved, or a slight improvement in efficiency. In what follows, we present a simple search cost model to study the impacts of consumer search cost on platform design, and further demonstrate the flexibility of the discriminatory access platform design to consider search costs.

\section{Impact of Search Costs on Platform Design}

The model and analysis presented thus far considers production costs but not other costs which can also be on the consumer side, and may be monetary, e.g., transportation and communication, or non-monetary, e.g., search costs. Platform designs center around lowering entry costs for producers, oftentimes leading to increased production and competition, and lowering search costs for consumers. In this section, we present a simple search cost model which highlights the ability of the discriminatory access design to also consider search costs, and the distinction between open access and discriminatory access designs.

As we are working in the networked Cournot setting where demand is aggregated, we must use a simple model for search costs. We define a search cost for each consumer market $j, r_{j}$, on the consumers as a product of their consumer surplus (taking care of the simple assuring assumption that markets are rational and that search costs can never be more than their surplus) and a discount factor $f(n)$ which monotonically increases with the number of firms $n$, and lastly, a market-specific parameter $\theta$ highlighting potentially different market segments with differing search costs, defined 
as follows:

$$
r_{j}=\theta f(n) C S_{j}, 0 \leq \theta \leq 1,
$$

where function $f$ has the following properties:

$$
f(1)=0, \lim _{n \rightarrow \infty} f(n)=1, f \text { monotonically increasing }
$$

This search cost model ensures that (i) search cost penalties cannot exceed consumer surplusan assumption that search cost can never cause the market to receive negative utility, (ii) differentiation can be made through $\theta$ for low and high search cost participants, and that (iii) search cost increases in the number of firms exposed to - the effort or cost has to increase as the number of choices increase.

Remark. Since controlled allocation platforms essentially make all decisions on allocation, the search cost is 0 for consumers but continue to maintain its previous negative result from Section 4, implying that search costs has no effect on controlled allocation platforms.

In light of the difficulty to analyze without a further parametric form of $f(n)$, we assume hereafter that $f(n)$ assumes the form of $f(n)=\frac{n-1}{n+1}$, fulfilling the preceding properties we listed.

Open access designs, by definition, presents all possible choices to consumers, allowing them to make production and allocation decisions, but also thereby incurring large search costs. In the following theorem, we highlight the impact of search costs on open access platforms.

Theorem 6.1. Open Access Platforms with search costs defined with $f(n)=\frac{n-1}{n+1}$ have worst case efficiency loss $\Omega(n)$.

On the other hand, referring to Corollary 5.1.1, where the price of anarchy is $\frac{4}{3}$ at $n=1$ (which coincidentally incurs zero search cost) for discriminatory access platform designs, they maintain its worst case constant bound price of anarchy under linear cost functions and search costs.

Prior to this section, it seems that the performance guarantees attained by open access and discriminatory access platform designs are similar. This section presents a preliminary look at the impact of search cost on platform design, in particular highlighting the potentially post-search unbounded worst case efficiency loss result for open access platforms, and the flexibility of the discriminatory access platform design to consider search costs. It remains important to study this further, e.g., to find out how to optimize network design with search costs, or how computation or other costs involved may affect the performance of discriminatory access designs too.

\section{Conclusion and Future Work}

Online platforms have emerged for different marketplaces over the last two decades. Even for a similar industry, platforms have varied designs, e.g., Uber vs. Didi, Amazon vs. eBay. These different designs compel a better comprehension of platform designs, and an understanding of the situations in which certain platform designs work well, and the difficulty involved in some of the more intricate designs.

In this work, we present an overview of platform designs related to access and allocation control, proving their respective performances with regards to preserving social welfare with respect to the optimal social welfare. The platform designs we consider aims to maximize social welfare obtained and operates at different levels of transparency and control under the networked Cournot model. 
Specifically, they include open access, controlled allocation and discriminatory access platform designs respectively.

Open access platforms, exemplified by eBay, allow all firms to interact transparently and participate freely in all markets. Under the setting considered in this work, these platforms designs preserves at least half of production and $2 / 3$ of the optimal social welfare in the worst case. We also sharpen this efficiency loss bound by considering how the efficiency loss in open access platforms is affected by the cost asymmetry among firms. However, under a simple model of search cost, we show that open access platforms can be unboundedly inefficient in the worst case after factoring in search costs, i.e., the worst case efficiency loss can grow linearly in the number of firms after consideration of search costs.

Controlled allocation platforms assign production to maximize social welfare, potentially causing a reduction in production from firms, as empirically witnessed under ride-sharing platforms with similar designs such as Uber. We show under the setting considered in this work, that it leads to unbounded inefficiency in the worst case, i.e., the price of anarchy can grow linearly in the number of markets. We extend the construction to optimizing over a convex combination of revenue and consumer surplus, which leads to different worst case efficiency loss bounds.

Discriminatory access platform designs, exemplified by Amazon's Buybox, optimize network access, to restrict the interactions of certain firm-market pairs. We show that discriminatory access platforms preserves at least 3/4 of the optimal social welfare even after the consideration of search costs. Additionally, while network design may be difficult in general, we design a greedy algorithm that performs network design efficiently under linear cost constraints, which we show to be the worst case cost functions.

This work sheds light on the tradeoffs between transparency, control and worst case efficiency loss. Moreover, the main contributing factors for high and low efficiency loss, e.g., misaligned objectives that lead to production withholding for controlled allocation platform designs, are indeed witnessed too in real-life online platforms. For example, empirical studies on Uber have shown that drivers collaborate to synchronize their productions to cause demand spikes, resulting in better individual payoff but decreased system performance.

Our results shed light on the differences between industries and their specific needs. For ridesharing platforms, the high search cost involved often leads to a controlled allocation platform design while marketplaces like Amazon have lower search cost and perform well under discriminatory access designs. Electricity markets are an important platform that work under physical network constraints and therefore compelled to be under a controlled allocation design, which may be inefficient. More work is needed to understand some of these issues, e.g., physical network constraints and the potential of demand management mechanisms such as demand response.

\section{References}

[1] M. Abolhassani, M. H. Bateni, M. Hajiaghayi, H. Mahini, and A. Sawant. Network cournot competition. In International Conference on Web and Internet Economics, pages 15-29. Springer, 2014.

[2] D. Abreu and M. Manea. Bargaining and efficiency in networks. Journal of Economic Theory, 147(1):43-70, 2012. 
[3] P. Afeche, Z. Liu, and C. Maglaras. Ride-hailing networks with strategic drivers: The impact of platform control capabilities on performance. 2018.

[4] N. Agarwal, I. Ashlagi, E. Azevedo, C. R. Featherstone, and Ö. Karaduman. Market failure in kidney exchange. Technical report, National Bureau of Economic Research, 2018.

[5] M. Akbarpour, S. Li, and S. Oveis Gharan. Thickness and information in dynamic matching markets. 2017. Available at SSRN: https://ssrn.com/abstract=2394319.

[6] R. Alijani, S. Banerjee, S. Gollapudi, K. Kollias, and K. Munagala. Two-sided facility location. arXiv preprint arXiv:1711.11392, 2017.

[7] H. Alsabah, B. Bernard, A. Capponi, G. Iyengar, and J. Sethuraman. Multiregional oligopoly with capacity constraints. SSRN Electronic Journal, 012019.

[8] E. Anshelevich and S. Sekar. Price competition in networked markets: How do monopolies impact social welfare? In Web and Internet Economics, pages 16-30. Springer, 2015.

[9] M. Armstrong. Competition in two-sided markets. The RAND Journal of Economics, 37(3):668-691, 2006.

[10] I. Ashlagi, M. Burq, P. Jaillet, and V. Manshadi. On matching and thickness in heterogeneous dynamic markets. Available at SSRN 3067596, 2018.

[11] J. Y. Bakos. Reducing buyer search costs: Implications for electronic marketplaces. Management science, 43(12):1676-1692, 1997.

[12] S. Banerjee, D. Freund, and T. Lykouris. Multi-objective pricing for shared vehicle systems. CoRR, abs/1608.06819, 2016.

[13] S. Banerjee, S. Gollapudi, K. Kollias, and K. Munagala. Segmenting two-sided markets. In Proceedings of the 26th International Conference on World Wide Web, pages 63-72, 2017.

[14] S. Banerjee, C. Riquelme, and R. Johari. Pricing in ride-share platforms: A queueing-theoretic approach. 2015. Available at SSRN: https://ssrn.com/abstract=2568258.

[15] K. Bimpikis, O. Candogan, and D. Saban. Spatial pricing in ride-sharing networks. 2016. Available at SSRN: https://ssrn.com/abstract $=2868080$.

[16] K. Bimpikis, S. Ehsani, and R. Ilkilic. Cournot competition in networked markets. In EC, page $733,2014$.

[17] S. Bose, D. W. Cai, S. Low, and A. Wierman. The role of a market maker in networked cournot competition. In 53rd IEEE Conference on Decision and Control, pages 4479-4484. IEEE, 2014.

[18] K. Boudreau. Open platform strategies and innovation: Granting access vs. devolving control. Management Science, 56(10):1849-1872, 2010.

[19] F. Branco, M. Sun, and J. M. Villas-Boas. Too much information? information provision and search costs. Marketing Science, 35(4):605-618, 2015. 
[20] D. Cai, S. Bose, and A. Wierman. On the role of a market maker in networked cournot competition. arXiv preprint arXiv:1701.08896, 2017.

[21] S. Chawla, J. D. Hartline, and R. Kleinberg. Algorithmic pricing via virtual valuations. In Proceedings of the 8th ACM conference on Electronic commerce, pages 243-251. ACM, 2007.

[22] S. Chawla, J. D. Hartline, D. L. Malec, and B. Sivan. Multi-parameter mechanism design and sequential posted pricing. In Proceedings of the forty-second ACM symposium on Theory of computing, pages 311-320. ACM, 2010.

[23] S. Chawla and T. Roughgarden. Bertrand competition in networks. In Algorithmic Game Theory, pages 70-82. Springer, 2008.

[24] J. Y. Chen. Thrown under the bus and outrunning it! the logic of didi and taxi drivers labour and activism in the on-demand economy. New Media \& Society, page 1461444817729149, 2017.

[25] L. Chen, A. Mislove, and C. Wilson. An empirical analysis of algorithmic pricing on amazon marketplace. In Proceedings of the 25th International Conference on World Wide Web, pages 1339-1349. International World Wide Web Conferences Steering Committee, 2016.

[26] L. Chen and C. Wilson. Observing algorithmic marketplaces in-the-wild. ACM SIGecom Exchanges, 15(2):34-39, 2017.

[27] A. Chircu and R. J. Kauffman. Digital intermediation in electronic commerce-the ebay model. E-commerce and V-business, pages 43-66, 2001.

[28] K. Diehl, L. J. Kornish, and J. G. Lynch Jr. Smart agents: When lower search costs for quality information increase price sensitivity. Journal of Consumer Research, 30(1):56-71, 2003.

[29] M. Dinerstein, L. Einav, J. Levin, and N. Sundaresan. Consumer price search and platform design in internet commerce. American Economic Review, 108(7):1820-59, 2018.

[30] L. Einav, T. Kuchler, J. Levin, and N. Sundaresan. Assessing sale strategies in online markets using matched listings. American Economic Journal: Microeconomics, 7(2):215-247, 2015.

[31] M. Elliott. Inefficiencies in networked markets. American Economic Journal: Microeconomics, $7(4): 43-82,2015$.

[32] D. S. Evans and R. Schmalensee. Matchmakers: The new economics of multisided platforms. Harvard Business Review Press, 2016.

[33] P. Evans and A. Gawer. The rise of the platform enterprise: A global survey, January 2016.

[34] Z. Fang, L. Huang, and A. Wierman. Loyalty programs in the sharing economy: Optimality and competition. arXiv preprint arXiv:1805.03581, 2018.

[35] Forbes. Global 2000: The worlds largest public companies, 2018.

[36] T. Gamp. Guided search. Working paper, 2016.

[37] B. Gross and A. Acquisti. Balances of power on ebay: Peers or unequals. In Workshop on Economics of Peer-to-peer systems. Citeseer, 2003. 
[38] C. L. Guzmán. Price competition on network. Technical report, 2011.

[39] A. Hagiu and J. Wright. Multi-sided platforms. International Journal of Industrial Organization, 43:162-174, 2015.

[40] J. Hall, C. Kendrick, and C. Nosko. The effects of ubers surge pricing: A case study. The University of Chicago Booth School of Business, 2015.

[41] F. Heylighen. Why is open access development so successful? stigmergic organization and the economics of information. arXiv preprint cs/0612071, 2006.

[42] B. F. Hobbs. Linear complementarity models of nash-cournot competition in bilateral and poolco power markets. IEEE Power Engineering Review, 21(5):63-63, 2001.

[43] X. Hui, M. Saeedi, Z. Shen, and N. Sundaresan. Reputation and regulations: evidence from ebay. Management Science, 62(12):3604-3616, 2016.

[44] R. Ilkilic. Cournot competition on a network of markets and firms. FEEM Working Paper, 2009. Available at SSRN: https://ssrn.com/abstract $=1443216$.

[45] W. Jing-Yuan and Y. Smeers. Spatial oligopolistic electricity models with cournot generators and regulated transmission prices. Operations Research, 47(1):102-112, 1999.

[46] R. Johari and J. N. Tsitsiklis. Efficiency loss in cournot games. Technical Report, 2005.

[47] Y. Kanoria and D. Saban. Facilitating the search for partners on matching platforms: Restricting agents' actions. Columbia Business School Research Paper No. 17-74, 2017. Available at SSRN: https://ssrn.com/abstract=3004814.

[48] E. Koutsoupias and C. H. Papadimitriou. Worst-case equilibria. In Symposium on Theoretical Aspects of Computer Science, pages 404-413, 1999.

[49] S. Lahiri and Y. Ono. Helping minor firms reduces welfare. The economic journal, 98(393):1199-1202, 1988.

[50] H. Le Cadre. On the efficiency of local electricity markets under decentralized and centralized designs: a multi-leader stackelberg game analysis. Central European Journal of Operations Research, pages 1-32, 2018.

[51] Y. Li, B. L. Ng, M. Trayer, and L. Liu. Automated residential demand response: Algorithmic implications of pricing models. IEEE Transactions on Smart Grid, 3(4):1712-1721, 2012.

[52] S. Liang, M. Schuckert, R. Law, and C.-C. Chen. Be a superhost: The importance of badge systems for peer-to-peer rental accommodations. Tourism management, 60:454-465, 2017.

[53] A. Lu, P. I. Frazier, and O. Kislev. Surge pricing moves uber's driver-partners. In Proceedings of the 2018 ACM Conference on Economics and Computation, pages 3-3. ACM, 2018.

[54] M. Luca. Designing online marketplaces: Trust and reputation mechanisms. Innovation Policy and the Economy, 17(1):77-93, 2017. 
[55] Z.-Q. Luo, J.-S. Pang, and D. Ralph. Mathematical programs with equilibrium constraints. Cambridge University Press, 1996.

[56] H. Ma, F. Fang, and D. C. Parkes. Spatio-temporal pricing for ridesharing platforms. arXiv preprint arXiv:1801.04015, 2018.

[57] B. J. Malin and C. Chandler. Free to work anxiously: Splintering precarity among drivers for uber and lyft. Communication, Culture $\&$ Critique, 10(2):382-400, 2017.

[58] N. G. Mankiw and M. D. Whinston. Free entry and social inefficiency. The RAND Journal of Economics, pages 48-58, 1986.

[59] M. Motalleb, A. Eshraghi, E. Reihani, H. Sangrody, and R. Ghorbani. A game-theoretic demand response market with networked competition model. In Power Symposium (NAPS), 2017 North American, pages 1-6. IEEE, 2017.

[60] F. Nava. Efficiency in decentralized oligopolistic markets. Journal of Economic Theory, $157: 315-348,2015$.

[61] T. Nguyen. Coalitional bargaining in networks. Operations Research, 63(3):501-511, 2015.

[62] M. Nishida and M. Remer. Lowering consumer search costs can lead to higher prices. Economics Letters, 162:1-4, 2018.

[63] C. Nosko and S. Tadelis. The limits of reputation in platform markets: An empirical analysis and field experiment. Technical report, National Bureau of Economic Research, 2015.

[64] J. Z. Pang, H. Fu, W. Lee, and A. Wierman. The efficiency of open access in platforms for networked cournot markets. In IEEE International Conference on Computer Communications, 2017.

[65] G. Parker and M. Van Alstyne. Innovation, openness, and platform control. Management Science, 2017.

[66] J.-C. Rochet and J. Tirole. Platform competition in two-sided markets. Journal of the european economic association, 1(4):990-1029, 2003.

[67] A. Rosenblat and L. Stark. Algorithmic labor and information asymmetries: A case study of ubers drivers. International Journal Of Communication, 2016. Available at SSRN: https://ssrn.com/abstract $=2686227$.

[68] M. Rysman. The economics of two-sided markets. Journal of Economic Perspectives, 23(3):125-43, 2009.

[69] N. Scheiber. How uber uses psychological tricks to push its drivers' buttons. The New York Times, 2, 2017.

[70] J. Schor. Debating the sharing economy. Journal of Self-Governance 8 S Management Economics, 4(3), 2016.

[71] K. Suzumura and K. Kiyono. Entry barriers and economic welfare. The Review of Economic Studies, 54(1):157-167, 1987. 
[72] S. Tadelis. Reputation and feedback systems in online platform markets. Annual Review of Economics, 8:321-340, 2016.

[73] E. G. Weyl. A price theory of multi-sided platforms. The American Economic Review, 100(4):1642-1672, 2010.

[74] Y. Xu, D. Cai, S. Bose, and A. Wierman. On the efficiency of networked stackelberg competition. In Information Sciences and Systems (CISS), 2017 51st Annual Conference on, pages 1-6. IEEE, 2017.

\section{A Open Access Platforms}

\section{A.1 Proof of Lemma 3.2}

Let $q^{\mathrm{NE}}(F \times M) \in \mathcal{Q}(F \times M)$ be the unique Nash equilibrium of the game $(F, \mathcal{Q}(F \times M), \pi)$ associated with an arbitrary cost function profile $C$. Throughout the proof, we always consider a networked Cournot game associated with the edge set $F \times M$. Thus, for notational simplicity we use $q^{\mathrm{NE}}$ instead of $q^{\mathrm{NE}}(F \times M)$ for the remainder of the proof. For each $i \in F$, we define the non-negative scalar $\lambda_{i}$ according to

$$
\lambda_{i}:=\partial^{+} C_{i}\left(\sum_{j=1}^{m} q_{i j}^{\mathrm{NE}}\right) .
$$

Here, $\lambda_{i}$ is the marginal cost of firm $i$ at the unique Nash equilibrium of the game $(F, \mathcal{Q}(F \times$ $M), \pi)$. We define a (piecewise) linear cost function profile $\bar{C}=\left(\bar{C}_{1}, \ldots, \bar{C}_{n}\right)$ according to

$$
\bar{C}_{i}\left(s_{i}\right):=\max \left\{\lambda_{i} s_{i}, 0\right\}, \quad i=1, \ldots, n .
$$

Clearly, $\bar{C}_{i}$ is convex, and differentiable on $(0, \infty)$ for each $i \in\{1, \ldots, n\}$. Additionally, the stationarity conditions of firms' profit maximization problem under the cost function profiles $C$ and $\bar{C}$ are identical at $q^{\mathrm{NE}}$. The combination of these two facts shows that $q^{\mathrm{NE}}$ is the unique Nash equilibrium of the game $(F, \mathcal{Q}(F \times M), \bar{\pi})$ associated with the cost function profile $\bar{C}$. Our objective is to show that $\rho(F \times M, C) \leq \rho(F \times M, \bar{C})$, i.e.,

$$
\frac{\mathrm{SW}\left(q^{\mathrm{NE}}, C\right)}{\mathrm{SW}^{*}(F \times M, C)} \geq \frac{\mathrm{SW}\left(q^{\mathrm{NE}}, \bar{C}\right)}{\mathrm{SW}^{*}(F \times M, \bar{C})} .
$$

In showing this, we first define the scalar $\mu_{i}$ for each firm $i \in F$ according to

$$
\mu_{i}:=\partial^{+} C_{i}\left(\sum_{j=1}^{m} q_{i j}^{\mathrm{NE}}\right) \cdot\left(\sum_{j=1}^{m} q_{i j}^{\mathrm{NE}}\right)-C_{i}\left(\sum_{j=1}^{m} q_{i j}^{\mathrm{NE}}\right) .
$$

For each firm $i, \mu_{i}$ equals the absolute difference in his production cost at Nash equilibrium associated with the cost function profiles $C$ and $\bar{C}$.

With the definition of $\mu_{i}$ in hand, we define an "intermediate" cost function profile $\widetilde{C}=$ $\left(\widetilde{C}_{1}, \ldots, \widetilde{C}_{n}\right)$ according to:

$$
\widetilde{C}_{i}\left(s_{i}\right):=\max \left\{\lambda_{i} s_{i}-\mu_{i}, 0\right\}, \quad i=1, \ldots, n .
$$


The cost function profile $\widetilde{C}$ makes the connection between the cost function profiles $C$ and $\bar{C}$. On one hand, for each firm $i \in F$, the cost function $\widetilde{C}_{i}$ can be regarded as a linearization of the original cost function $C_{i}$ around the Nash equilibrium of the game $(F, \mathcal{Q}(F \times M), \pi)$. On the other hand, it can be interpreted as a translation of the cost function $\bar{C}_{i}$ downwards along the y-axis of length $\mu_{i}$, while keeping the resulting cost function non-negative for all real numbers. Note that the stationarity conditions of firms' profit maximization problem under the cost function profiles $C$ and $\widetilde{C}$ are identical at $q^{\mathrm{NE}}$. It follows that $q^{\mathrm{NE}}$ is the unique Nash equilibrium of the game $(F, \mathcal{Q}(F \times M), \widetilde{\pi})$ associated with the cost function profile $\widetilde{C}$.

Since firms' production costs at $q^{\mathrm{NE}}$ are equal for the cost function profiles $C$ and $\widetilde{C}$, we have that $\mathrm{SW}\left(q^{\mathrm{NE}}, C\right)=\mathrm{SW}\left(q^{\mathrm{NE}}, \widetilde{C}\right)$. Moreover, since $C_{i}\left(s_{i}\right) \geq \widetilde{C}_{i}\left(s_{i}\right)$ for all $s_{i} \in \mathbb{R}_{+}$, we have that $\mathrm{SW}^{*}(F \times M, C) \leq \mathrm{SW}^{*}(F \times M, \widetilde{C})$. It follows that

$$
\frac{\mathrm{SW}\left(q^{\mathrm{NE}}, C\right)}{\mathrm{SW}^{*}(F \times M, C)} \geq \frac{\mathrm{SW}\left(q^{\mathrm{NE}}, \widetilde{C}\right)}{\mathrm{SW}^{*}(F \times M, \widetilde{C})} .
$$

Additionally, the previous translation from $\bar{C}$ to $\widetilde{C}$ implies that $S W\left(q^{\mathrm{NE}}, \widetilde{C}\right)$ and $S W\left(q^{\mathrm{NE}}, \bar{C}\right)$ are related according to:

$$
\mathrm{SW}\left(q^{\mathrm{NE}}, \bar{C}\right)=\mathrm{SW}\left(q^{\mathrm{NE}}, \widetilde{C}\right)-\sum_{i=1}^{n} \mu_{i} \geq 0 .
$$

We claim that the following inequality holds for the efficient social welfare $\mathrm{SW}^{*}(F \times M, \bar{C})$ associated with the cost function profile $\bar{C}$ :

$$
\mathrm{SW}^{*}(F \times M, \bar{C}) \geq \mathrm{SW}^{*}(F \times M, \widetilde{C})-\sum_{i=1}^{n} \mu_{i} .
$$

To see this, let $q^{*}$ be an efficient supply profile under the cost function profile $\widetilde{C}$. For each $i \in F$, we have

$$
\bar{C}_{i}\left(\sum_{j=1}^{m} q_{i j}^{*}\right) \leq \widetilde{C}_{i}\left(\sum_{j=1}^{m} q_{i j}^{*}\right)+\mu_{i}
$$

This inequality implies that

$$
\begin{aligned}
\mathrm{SW}^{*}(F \times M, \bar{C}) & \geq \mathrm{SW}\left(q^{*}, \bar{C}\right) \geq \mathrm{SW}\left(q^{*}, \widetilde{C}\right)-\sum_{i=1}^{n} \mu_{i} \\
& =\mathrm{SW}^{*}(F \times M, \widetilde{C})-\sum_{i=1}^{n} \mu_{i},
\end{aligned}
$$

The above inequality, in combination with inequalities Equations 13 and 14, shows that

$$
\begin{aligned}
& \frac{\mathrm{SW}\left(q^{\mathrm{NE}}, C\right)}{\mathrm{SW}^{*}(F \times M, C)} \geq \frac{\mathrm{SW}\left(q^{\mathrm{NE}}, \widetilde{C}\right)}{\mathrm{SW}^{*}(F \times M, \widetilde{C})} \\
& \quad \geq \frac{\mathrm{SW}\left(q^{\mathrm{NE}}, \widetilde{C}\right)-\sum_{i=1}^{n} \mu_{i}}{\mathrm{SW}^{*}(F \times M, \widetilde{C})-\sum_{i=1}^{n} \mu_{i}} \geq \frac{\mathrm{SW}\left(q^{\mathrm{NE}}, \bar{C}\right)}{\mathrm{SW}^{*}(F \times M, \bar{C})},
\end{aligned}
$$

as needed to be shown. 


\section{A.2 Proof of Proposition 3.1}

It follows from Lemma 3.2 that the worst symmetric cost function profile that maximizes $\rho(F \times$ $M, C)$ consists of $n$ identical cost functions that are linear on $(0, \infty)$. Thus, to upper bound the PoA of the networked Cournot game, it suffices to consider a cost function profile $\bar{C}$ that satisfies

$$
\bar{C}_{i}(x)=c x \quad \text { for } x \geq 0, \quad \text { for all } i \in F,
$$

for a finite positive constant $c>0$ that is independent of $i$.

Given the assumption on the (piecewise) linearity of cost, it is straightforward to show that the unique Nash equilibrium and an efficient supply profile of the corresponding networked Cournot game are given by

$$
q_{i j}^{\mathrm{NE}}=\frac{\left(\alpha_{j}-c\right)^{+}}{\beta_{j}(n+1)}, \quad \text { and } \quad q_{i j}^{*}=\frac{\left(\alpha_{j}-c\right)^{+}}{\beta_{j} n},
$$

respectively, for each $i \in F, j \in M$. It follows that the social welfare at the unique Nash equilibrium $q^{\mathrm{NE}}$ of this Cournot game is given by

$$
S W^{*}\left(q^{\mathrm{NE}}, \bar{C}\right)=\sum_{j=1}^{m} \frac{\left(\left(\alpha_{j}-c\right)^{+}\right)^{2}}{2 \beta_{j}}\left(1-\frac{1}{(n+1)^{2}}\right) .
$$

And the efficient social welfare is given by

$$
S W^{*}(F \times M, \bar{C})=\sum_{j=1}^{m} \frac{\left(\left(\alpha_{j}-c\right)^{+}\right)^{2}}{2 \beta_{j}} .
$$

Hence, the price of anarchy associated with the cost function profile $\bar{C}$ is given by

$$
\rho(F \times M, \bar{C})=\left\{\begin{array}{ll}
1+\frac{1}{(n+1)^{2}-1} & \text { if } \max _{j \in M} \alpha_{j}>c, \\
1 & \text { otherwise }
\end{array} .\right.
$$

Choosing $c<\max _{j \in M} \alpha_{j}$ gives the worst-case cost function profile that maximizes the price of anarchy over symmetric cost function profiles. This completes the proof.

\section{A.3 Proof of Theorem 3.1}

It follows from Lemma 3.2 that the worst cost function profile that maximizes $\rho(F \times M, C)$ consists of cost functions that are linear on $(0, \infty)$. We thus assume that each firm's cost function satisfies $\bar{C}_{i}\left(s_{i}\right)=\left(c_{i} s_{i}\right)^{+}$, for $i=1, \ldots, n$, where we assume without loss of generality that $c_{1} \leq \cdots \leq c_{n}$.

Note that, for the case in which $n=1$, a direct application of Proposition 3.1] provides a tight price of anarchy bound of $\rho(F \times M, C) \leq 4 / 3$. For the remainder of the proof, we restrict ourselves to $n \geq 2$.

We first consider the simple setting where the number of markets $m=1$. That is, the set of markets $M=\{1\}$. Without loss of generality, we assume that $c_{1}<\alpha_{1}$. Since cost functions are linear, there exists an efficient supply profile $q^{*}$ that assigns all production to firm 1, i.e., 
$q_{i 1}^{*}=0$ for $i=2, \ldots, n$. The supply from firm 1 and the corresponding efficient social welfare are given by:

$$
q_{11}^{*}=\frac{\alpha_{1}-c_{1}}{\beta_{1}}, \quad \text { and } \quad S W^{*}(F \times\{1\}, \bar{C})=\frac{\left(\alpha_{1}-c_{1}\right)^{2}}{2 \beta_{1}} .
$$

Fixing $\alpha_{1}, \beta_{1}, c_{1}$, we can optimize over $c_{2}, \ldots, c_{n}$ in order to minimize the social welfare at the unique Nash equilibrium of the Cournot game. Using similar arguments as in the proof of Theorem 12 in [46], one can formulate this problem as a symmetric convex program over the production quantities at Nash equilibrium of the remaining firms. The optimal value of $c_{2}, \ldots, c_{n}$ is given by

$$
c_{i}^{*}=\alpha_{1}-\frac{2 n+3}{3 n+5}\left(\alpha_{1}-c_{1}\right), \quad \text { for } i=2, \ldots, n .
$$

And the production quantity of each producer is given by

$$
q_{i 1}= \begin{cases}\frac{\alpha_{1}-c_{1}}{\beta_{1}} \cdot \frac{n+3}{3 n+5} & \text { if } i=1 . \\ \frac{\alpha_{1}-c_{1}}{\beta_{1}} \cdot \frac{1}{3 n+5}, & \text { if } i=2, \ldots, n .\end{cases}
$$

Define the cost function profile $\bar{C}^{*}=\left(\bar{C}_{1}^{*}, \ldots, \bar{C}_{n}^{*}\right)$ according to $\bar{C}_{1}^{*}\left(s_{1}\right)=\left(c_{1} s_{1}\right)^{+}$and $\bar{C}_{i}^{*}\left(s_{i}\right)=$ $\left(c_{i}^{*} s_{i}\right)^{+}$for $i=2, \ldots, n$. Thus, for the fixed parameters $\alpha_{1}, \beta_{1}, c_{1}$, the minimum social welfare at Nash equilibrium is given by

$$
\mathrm{SW}\left(q^{\mathrm{NE}}(F \times\{1\}), \bar{C}^{*}\right)=\frac{(n+2)\left(\alpha_{1}-c_{1}\right)^{2}}{(3 n+5) \beta_{1}} .
$$

It follows that for any linear cost function profile $\bar{C}$, we have

$$
\rho(F \times\{1\}, \bar{C}) \leq \rho\left(F \times\{1\}, \bar{C}^{*}\right)=\frac{3}{2}\left(1-\frac{1}{3 n+6}\right) .
$$

We now consider the slightly more complicated setting in which $m>1$. The efficient social welfare associated with a linear cost function profile $\bar{C}$ is given by

$$
\mathrm{SW}^{*}(F \times M, \bar{C})=\sum_{j=1}^{m} \frac{\left(\left(\alpha_{j}-c_{1}\right)^{+}\right)^{2}}{2 \beta_{j}} .
$$

Given the linearity of firms' cost functions over $(0, \infty)$, the networked Cournot game decouples across markets. Thus, the social welfare at Nash equilibrium satisfies

$$
\begin{gathered}
\operatorname{SW}\left(q^{\mathrm{NE}}(F \times M), \bar{C}\right)=\sum_{j=1}^{m} \operatorname{SW}\left(q^{\mathrm{NE}}(F \times\{j\}), \bar{C}\right) \\
\geq \sum_{j=1}^{m} \frac{\left(\left(\alpha_{j}-c_{1}\right)^{+}\right)^{2}}{3 \beta_{j}\left(1-\frac{1}{3 n+6}\right)}=\frac{\mathrm{SW}^{*}(F \times M, \bar{C})}{\frac{3}{2}\left(1-\frac{1}{3 n+6}\right)} .
\end{gathered}
$$

It follows that the price of anarchy $\rho(F \times M, \bar{C})$ satisfies

$$
\rho(F \times M, \bar{C}) \leq \frac{3}{2}\left(1-\frac{1}{3 n+6}\right) .
$$

Additionally, it is straightforward to check that this price of anarchy bound is achieved by the cost function profile $\bar{C}^{*}$ if $\alpha_{1}=\alpha_{2}=\cdots=\alpha_{m}$. This completes the proof. 


\section{A.4 Proof of Proposition 3.2}

We only provide a proof of the price of anarchy bound, since the proof on its tightness is straightforward. The key intuition of the proof is that, under a linear cost function profile, the networked Cournot game decouples across markets. The proof proceeds in two parts.

Part 1: We provide a price of anarchy bound for the case in which the number of markets $m=1$. Namely, for any cost function profile $C \in \mathcal{L}^{n}\left(c_{\min }, c_{\max }\right)$, we have

$$
\rho(F \times\{j\}, C) \leq \frac{1}{\frac{2 n+4}{3 n+5}+\delta\left(\gamma_{j}, n\right)}, \quad j=1, \ldots, m .
$$

We omit the proof of this price of anarchy bound, as it follows from similar arguments as in the proof of Theorem 3.1 .

Part 2: We provide a price of anarchy bound for the case in which the number of market $m>1$. Let the cost function of each firm $i \in F$ be given by

$$
C_{i}\left(s_{i}\right)=\left(c_{i} s_{i}\right)^{+}, \quad \text { where } c_{i} \in\left[c_{\min }, c_{\max }\right] .
$$

Without loss of generality, we assume that $c_{1} \leq \cdots \leq c_{n}$. Thus, the efficient social welfare associated with the edge set $F \times M$ and the cost function profile $C$ is given by

$$
\mathrm{SW}^{*}(F \times M, C)=\sum_{j=1}^{m} \frac{\left(\left(\alpha_{j}-c_{1}\right)^{+}\right)^{2}}{2 \beta_{j}} .
$$

Since firms' cost functions are linear on $(0, \infty)$, the networked Cournot game decouples across markets. It follows that the social welfare at the unique Nash equilibrium of the game $(F, \mathcal{Q}(F \times$ $M), \pi)$ satisfies

$$
\mathrm{SW}\left(q^{\mathrm{NE}}(F \times M), C\right)=\sum_{j=1}^{m} \mathrm{SW}\left(q^{\mathrm{NE}}(F \times\{j\}), C\right) .
$$

Additionally, the term $\mathrm{SW}\left(q^{\mathrm{NE}}(F \times\{j\}), C\right)$ satisfies

$$
\begin{aligned}
\mathrm{SW} & \left(q^{\mathrm{NE}}(F \times\{j\}), C\right)=\frac{\mathrm{SW}^{*}(F \times\{j\}, C)}{\rho(F \times\{j\}, C)} \\
& \geq \sum_{j=1}^{m} \frac{\left(\left(\alpha_{j}-c_{1}\right)^{+}\right)^{2}}{2 \beta_{j}}\left(\frac{2 n+4}{3 n+5}+\delta\left(\gamma_{j}, n\right)\right) .
\end{aligned}
$$

Here, equation 17 follows from the definition of the price of anarchy, and inequality 18 follows from the price of anarchy bound in Step 1. A combination of Eq. (16) and inequality (18) provides the following lower bound on the reciprocal of the price of anarchy $\rho(F \times M, C)$ :

$$
\frac{1}{\rho(F \times M, C)} \geq \frac{\sum_{j=1}^{m}\left(\frac{2 n+4}{3 n+5}+\delta\left(\gamma_{j}, n\right)\right) \frac{\left(\left(\alpha_{j}-c_{1}\right)^{+}\right)^{2}}{\beta_{j}}}{\sum_{j=1}^{m} \frac{\left(\left(\alpha_{j}-c_{1}\right)^{+}\right)^{2}}{\beta_{j}}} .
$$

One can verify that the partial derivative of the right-hand-side (RHS) of the above inequality with respect to $c_{1}$ is non-negative for $c_{1} \in\left[c_{\min }, c_{\max }\right]$. Hence, choosing $c_{1}=c_{\min }$ minimizes the RHS of the above inequality. This completes the proof. 
Lemma A.1. There exists firm $i$, such that $s_{i}^{N} \leq s_{i}^{*}$.

Proof. Proof: Suppose not. Then $\forall i, s_{i}^{N}>s_{i}^{*}$, the following two cases are possible. First, denote the sets $S_{N}$ and $S_{*}$ the sets $\left\{j: d_{j}^{N}>0\right\}$ and $\left\{j: d_{j}^{*}>0\right\}$.

Case 1: $S_{N} \subseteq S_{*}$ :

$$
s_{i}^{N}>s_{i}^{*} \Rightarrow \exists j \in S_{N} \text { s.t. } d_{j}^{N}>d_{j}^{*} \Rightarrow p_{j}^{*}>p_{j}^{N},
$$

but considering any firm $i$ such that $q_{i j}^{N}>0$,

$$
\alpha_{j}>p_{j}^{N}=c_{i}^{\prime}\left(s_{i}^{N}\right)+\beta_{j} q_{i j}^{N}>c_{i}^{\prime}\left(s_{i}^{N}\right)>c_{i}^{\prime}\left(s_{i}^{*}\right)
$$

but by the optimality of $*$,

$$
c_{i}^{\prime}\left(s_{i}^{*}\right) \geq p_{j}^{*} \Rightarrow p_{j}^{N}>p_{j}^{*}
$$

a contradiction.

Case 2: $\exists$ market $j$ s.t. $j \in S_{N} \backslash S_{*}$.

For any $i$ such that $q_{i j}^{N}>0, c_{i}^{\prime}\left(s_{i}^{N}\right)<\alpha_{j} \leq c_{i}^{\prime}\left(s_{i}^{*}\right)$, a contradiction to $s_{i}^{N}>s_{i}^{*}$.

Proof. Proof of Lemma 3.1. Suppose on the other hand that there is a market $j$ such that $d_{j}^{N}<d_{j}^{*}$. We show that this contradicts the preceding lemma. For any firm $i$ such that $q_{i j}^{N}=0$, we have $c_{i}^{\prime}\left(s_{i}^{N}\right) \geq p_{j}^{N} \geq p_{j}^{*}=c_{i}^{\prime}\left(s_{i}^{*}\right)$, and by monotonicity, $s_{i}^{N}>s_{i}^{*}$. On the other hand, if $q_{i j}^{N}>0$,

$$
c_{i}^{\prime}\left(s_{i}^{N}\right)=\alpha_{j}-\beta_{j} d_{j}^{N}-\beta_{j} q_{i j}^{N}>\alpha_{j}-\beta_{j} \frac{d_{j}^{*}}{2}-\beta_{j} \frac{d_{j}^{*}}{2}=\alpha_{j}-\beta_{j} d_{j}^{*}=c_{i}^{\prime}\left(s_{i}^{*}\right),
$$

again implying that $s_{i}^{N}>s_{i}^{*}$ for all $i$, a contradiction to the preceding lemma.

\section{B Proofs from Section 4}

Before providing a proof of Theorem 4.1 we first state and prove the key structural lemma we use in the proof.

Lemma B.1. For a firm with costless production, a set of linear demand markets under controlled allocation is equivalent to a single market with a convex, piecewise linear demand curve. Conversely, any convex, decreasing, piecewise linear demand curve with finitely many linear segments can be realized by a set of linear demand markets under controlled access.

Proof. Proof of Lemma B.1. The characterization of the socially optimal production, with $s$ fixed highlights that the platform will reallocate this amount to $d_{1}, \ldots, d_{m}$ such that $\sum_{j} d_{j}=s$, and for each market $j$ where $d_{j}>0, p_{j}$ is equivalent to a fixed price $p$ across markets; for each market $j$ where $d_{j}=0$, it must be that $\alpha_{j} \leq p$. This shows that, as $s$ increases, the allocation will enter the markets one by one in the order in which $\alpha_{j}$ decreases.

We say a market becomes active when supply starts entering it. For a set of active markets, before the next market becomes active, the marginal increase in supply will be allocated in proportion to $1 / \beta_{j}$ (in order to keep the prices the same). This fully describes the behavior of the platform.

Without loss of generality, assume the markets are ordered such that $\alpha_{1} \geq \ldots \geq \alpha_{m}$. From the firm's point of view, the platform is equivalent to a single market with a piecewise linear demand 
curve: when the price is between $\alpha_{1}$ and $\alpha_{2}$, the rate at which price drops when $s$ increases is $\beta_{1}$; for $p \in\left[\alpha_{2}, \alpha_{3}\right]$, the rate is $1 /\left(\frac{1}{\beta_{1}}+\frac{1}{\beta_{2}}\right)$. In general, when the first $k$ markets are active, prices drop $i$ at the rate of $\left(\sum_{j=1}^{k} \frac{1}{\beta_{j}}\right)^{-1}$. We call this single demand curve the aggregate demand curve.

For a given production level, the area under the aggregate demand curve is equal to the welfare in the original markets. The aggregate demand curve fully characterizes the set of markets under controlled access. Note that whenever a new market joins, the rate at which price drops becomes slower, therefore the aggregate demand curve is always convex.

Conversely, one can show that any convex, decreasing, piecewise linear demand curve consisting of finitely many linear segments is equivalent to a set of linear demand markets under controlled access.

Proof. Proof of Theorem 4.1. By Lemma B.1, we can focus on constructing an aggregate demand curve. Fix a constant $\lambda \in\left(0, \frac{1}{2}\right)$. The aggregate demand curve we construct for $m$ markets, $(m \geq 2)$, is

$$
p(d)=\max _{0 \leq h<m}\left(\lambda^{h}-\lambda^{2 h} d\right) .
$$

It is not hard to verify that this is the piecewise linear function that connects the following points: $(0,1),\left(\frac{1}{1+\lambda}, \frac{\lambda}{1+\lambda}\right),\left(\frac{1}{\lambda(1+\lambda)}, \frac{\lambda^{2}}{1+\lambda}\right), \cdots,\left(\frac{1}{\lambda^{m-2}(1+\lambda)}, \frac{\lambda^{m-1}}{1+\lambda}\right),\left(\frac{1}{\lambda^{m-1}}, 0\right)$. Being the maximum of a family of decreasing linear functions, $p(d)$ is obviously a convex decreasing function. We first calculate the optimal social welfare, the area under $p(d)$. The trapezoid whose vertices are $\left(\frac{1}{\lambda^{h-1}(1+\lambda)}, 0\right)$, $\left(\frac{1}{\lambda^{h-1}(1+\lambda)}, \frac{\lambda^{h}}{1+\lambda}\right),\left(\frac{1}{\lambda^{h}(1+\lambda)}, 0\right),\left(\frac{1}{\lambda^{h}(1+\lambda)}, \frac{\lambda^{h+1}}{1+\lambda}\right)$ has area

$$
\frac{1}{2}\left(\frac{1}{\lambda^{h}(1+\lambda)}-\frac{1}{\lambda^{h-1}(1+\lambda)}\right)\left(\frac{\lambda^{h}}{1+\lambda}+\frac{\lambda^{h+1}}{1+\lambda}\right)=\frac{1-\lambda}{1+\lambda} .
$$

There are $m-2$ such trapezoids under $p(d)$, and therefore the socially optimal welfare is $\Omega(m)$. On the other hand, the linear components of $p(d)$ are designed so that producing on any of the linear segment gives a maximal profit of $\frac{1}{4}$ (for the $h$-th segment, the profit maximizing production level is $\left.\frac{1}{2 \lambda^{h-1}}\right)$. The firm is indifferent to best responding to any of the linear segments, and all the production levels $\frac{1}{2 \lambda^{h}}$ for $h=0, \ldots, m-1$ are Nash equilibria. If we push the starting point of $p(d)$ from $(0,1)$ to $(0,1+\epsilon)$ for some $\epsilon>0$, then producing $\frac{1+\epsilon}{2}$ (by responding only to the first market) will be the unique equilibrium, resulting in a social welfare of only $3(1+\epsilon)^{2} / 8$. Therefore the price of anarchy is $\Omega(m)$.

Proof. Proof of Theorem 4.2.

In the $\sigma \leq 0$ regime, a similar example to the consumer surplus case would work. For example, if $\alpha_{1}=\alpha_{2}$, with $\beta_{1}=0$ and $\beta_{2}=\epsilon$, then the platform will assign all of the allocations to the second market. Consider a linear cost function $c=\left(\alpha_{1}-\epsilon\right) x$, then the optimal is to fulfill all of market 1 which gives us infinitely large social welfare, whereas the platform forces the firm to go to market 2 , which results in a constant social welfare.

On the other hand, when $\sigma \in(0,1]$, we can design a series of markets for a costless firm with a resulting price of anarchy that grows in the number of markets accessed, similar to the social welfare maximizing platform $(\lambda=0.5$ case $)$. Lastly, in the $\sigma>1$ regime, our price of anarchy 
bound decreases gracefully as the market maker objective approaches revenue maximizing, up till a point where it is worst to set up an open access platform, explaining the max operator.

We first show that regardless of the platform objective, the firms produce on aggregate a quantity equivalent to the quantity that maximizes revenue:

Lemma B.2. Regardless of the parameter $\sigma$ for one costless firm, optimal aggregate production in the platform including markets $M$ maximizes social welfare, i.e. $q^{*}=\sum_{j \in M} \frac{\alpha_{j}}{2 \beta_{j}}$.

Proof. Proof of Lemma B.2. Since market $m$ is included, then the markets are allocated at least the following amount of quantity before the last market is accessed:

$$
d_{j}=\frac{\alpha_{j}-\alpha_{m}}{\sigma \beta_{j}}
$$

Further, we know that any additional quantity introduced into the platform will be assigned $e_{j}$ to the respective markets respecting the following condition:

$$
\beta_{j} e_{j}=\beta_{m} e_{m} \Rightarrow e_{j}=\frac{\frac{1}{\beta_{j}}}{\sum_{k} \frac{1}{\beta_{k}}} Q
$$

Optimizing over the additional quantity $e_{j}$, the firm's profit can be written:

$$
\sum_{j=1}^{m}\left(\frac{\alpha_{j}-\alpha_{m}}{\sigma \beta_{j}}+e_{j}\right)\left(\alpha_{j}-\beta_{j}\left(\frac{\alpha_{j}-\alpha_{m}}{\sigma \beta_{j}}+e_{j}\right)\right)
$$

but with $e_{j}$ defined above and letting $A=\sum_{k} \frac{1}{\beta_{k}}$, this can be rewritten as:

$$
\sum_{j=1}^{m}\left(\frac{\alpha_{j}-\alpha_{m}}{\sigma \beta_{j}}+\frac{Q}{A \beta_{j}}\right)\left(\alpha_{j}-\frac{\alpha_{j}-\alpha_{m}}{\sigma}-\frac{Q}{A}\right)
$$

Differentiating with respect to $Q$, we have:

$$
\sum_{j=1}^{m}\left[\frac{1}{A \beta_{j}}\left(\alpha_{j}-\frac{\alpha_{j}-\alpha_{m}}{\sigma}-\frac{Q}{A}\right)+\left(\frac{\alpha_{j}-\alpha_{m}}{\sigma \beta_{j}}+\frac{Q}{A \beta_{j}}\right)\left(-\frac{1}{A}\right)\right]=0
$$

which gives us that:

$$
Q=\sum_{j=1}^{m}\left(\frac{\alpha_{j}}{2 \beta_{j}}-\frac{\alpha_{j}-\alpha_{m}}{\sigma \beta_{j}}\right)
$$

which implies that the optimal aggregate production is indeed $\sum_{j=1}^{m} \frac{\alpha_{j}}{2 \beta_{j}}$.

We propose a set of parameters for each market as in the social welfare maximizing case and prove that the inefficiency in these markets are reflected as in Theorem 4.2.

Market parameters are designed in the following form, similar to that of the social welfare maximizing scenario:

$$
\alpha_{1}=1, \beta_{1}=1, \& \alpha_{j}=\frac{a^{j-1} \theta^{j-1}}{1+\theta}, \beta_{j}=\frac{\theta^{2 j-2}}{1-\theta^{2}}, \forall j \geq 2,
$$


then if we can show that the (single costless) firm wants to stay in the first market only region, then the price of anarchy grows as a function of the sum of a geometric progression with ratio $a$, and thereby can be written:

$$
\rho=\frac{4}{3}\left(1+\frac{1-\theta}{1+\theta}\left(a^{2}+a^{4}+\ldots\right)\right)=\frac{4}{3}\left(1+\frac{(1-\theta) a^{2}}{(1+\theta)\left(1-a^{2}\right)}\right) \rightarrow_{\theta \rightarrow 0} \frac{4}{3}\left(\frac{1}{1-a^{2}}\right)
$$

The optimal revenue considering only the first market is $\frac{\alpha_{1}^{2}}{4 \beta_{1}}=\frac{1}{4}$. After some algebra, the amount allocated to market $j$ in the presence of $m$ many markets of the form defined above, or $d_{j, m}$, can be expressed as:

$$
\left(\left(\frac{1}{2}-\frac{1}{\sigma}\right) \sum_{i=1}^{m} \frac{\alpha_{i}}{\beta_{i}}\right) \frac{\frac{1}{\beta_{j}}}{\sum_{i=1}^{m} \frac{1}{\beta_{i}}}+\frac{\alpha_{j}}{\sigma \beta_{j}}
$$

and the prices at the markets then can be computed via $\alpha_{j}-\beta_{j} d_{j, m}$ :

$$
\left(1-\frac{1}{\sigma}\right) \alpha_{j}-\frac{1}{\sum_{i=1}^{m} \frac{1}{\beta_{i}}}\left(\left(\frac{1}{2}-\frac{1}{\sigma}\right) \sum_{i=1}^{m} \frac{\alpha_{i}}{\beta_{i}}\right)
$$

and thereby, revenue from market $j$ can be written:

$$
\left(\frac{1}{2}-\frac{1}{\sigma}\right) \frac{\sum_{i=1}^{m} \frac{\alpha_{i}}{\beta_{i}}}{\sum_{i=1}^{m} \frac{1}{\beta_{i}}}\left(1-\frac{2}{\sigma}\right) \frac{\alpha_{j}}{\beta_{j}}+\left(1-\frac{1}{\sigma}\right) \frac{\alpha_{j}^{2}}{\sigma \beta_{j}}-\frac{1}{\beta_{j}}\left(\frac{1}{\sum_{i=1}^{m} \frac{1}{\beta_{i}}}\right)^{2}\left(\frac{1}{2}-\frac{1}{\sigma}\right)^{2}\left(\sum_{i=1}^{m} \frac{\alpha_{i}}{\beta_{i}}\right)^{2}
$$

and revenue from all markets can be written:

$$
\left(\frac{1}{2}-\frac{1}{\sigma}\right)^{2} \frac{\left(\sum_{i=1}^{m} \frac{\alpha_{i}}{\beta_{i}}\right)^{2}}{\sum_{i=1}^{m} \frac{1}{\beta_{i}}}+\frac{1}{\sigma}\left(1-\frac{1}{\sigma}\right) \sum_{i=1}^{m} \frac{\alpha_{i}^{2}}{\beta_{i}}
$$

and we wish to show that $\forall \sigma$, we can find $a$ and $\theta$ such that the above aggregate revenue is less than the revenue at only the first market. (Recall that when $\sigma=1$, the second term is gone and the first term is the revenue from the social welfare case, and taking $a=1$, we retrieve the same righthand side as the social welfare case too, which equates to the revenue at the first market, i.e., 1/4. )

Lemma B.3. When $a=1$ and $\sigma=1$, then for any $\lambda \in(0,0.5)$,

$$
\left(\frac{1}{2}-\frac{1}{\sigma}\right)^{2} \frac{\left(\sum_{i=1}^{m} \frac{\alpha_{i}}{\beta_{i}}\right)^{2}}{\sum_{i=1}^{m} \frac{1}{\beta_{i}}}=\frac{1}{4} \Longleftrightarrow \frac{\left(\sum_{i=1}^{m} \frac{\alpha_{i}}{\beta_{i}}\right)^{2}}{\sum_{i=1}^{m} \frac{1}{\beta_{i}}}=1 .
$$

Proof. Proof of Lemma B.3.

This is proved under the social welfare maximizing platform, and restated here for convenience.

Another important and interesting point to note is that the two coefficients in the revenue term sum up to $1 / 4$, i.e.,

$$
\left(\frac{1}{2}-\frac{1}{\sigma}\right)^{2}+\frac{1}{\sigma}\left(1-\frac{1}{\sigma}\right)=\frac{1}{4} .
$$

We use this identity to prove the following lemma: 
Lemma B.4. For $\sigma \in(0,1]$, take $a=1$, and $\theta<0.5$, then

$$
\left(\frac{1}{2}-\frac{1}{\sigma}\right)^{2} \frac{\left(\sum_{i=1}^{m} \frac{\alpha_{i}}{\beta_{i}}\right)^{2}}{\sum_{i=1}^{m} \frac{1}{\beta_{i}}}+\frac{1}{\sigma}\left(1-\frac{1}{\sigma}\right) \sum_{i=1}^{m} \frac{\alpha_{i}^{2}}{\beta_{i}}<\frac{1}{4}
$$

Proof. Proof of Lemma B.4: From above, it remains to show that $\sum_{i=1}^{m} \frac{\alpha_{i}^{2}}{\beta_{i}} \geq 1$, but the first term in the sum is already 1 while the rest are nonnegative so we are done.

We now move to prove the case whereby $\sigma>1$, through the following lemma:

Lemma B.5. For $\sigma>1$, and taking $\theta$ small enough,

$$
\frac{\left(\sum_{i=1}^{m} \frac{\alpha_{i}}{\beta_{i}}\right)^{2}}{\sum_{i=1}^{m} \frac{1}{\beta_{i}}} \leq a^{2}
$$

This can be seen by considering the $\sum_{i=1}^{m} \frac{\alpha_{i}}{\beta_{i}}$ part and extracting $a$ from each summand. With this result, we can show the following:

Lemma B.6. For $\sigma>1, a \leq \sqrt{1-2 \sqrt{\frac{1}{\sigma}\left(1-\frac{1}{\sigma}\right)}}$ and taking $\lambda$ small enough, then

$$
\left(\frac{1}{2}-\frac{1}{\sigma}\right)^{2} \frac{\left(\sum_{i=1}^{m} \frac{\alpha_{i}}{\beta_{i}}\right)^{2}}{\sum_{i=1}^{m} \frac{1}{\beta_{i}}}+\frac{1}{\sigma}\left(1-\frac{1}{\sigma}\right) \sum_{i=1}^{m} \frac{\alpha_{i}^{2}}{\beta_{i}}<\frac{1}{4} .
$$

The proof of Theorem 4.2 follows from the above lemmas, and the PoA bounds follow.

\section{Discriminatory Access Platforms}

\section{C.1 Proof of Theorem $[5.2$}

Without loss of generality, we assume that $c_{1} \leq \cdots \leq c_{n}$. We only provide a proof for the case in which the number of market $m=1$, as it is straightforward to generalize our proof to the case in which $m>1$ under the assumption on the linearity of firms' cost functions.

We denote by $F_{1}(\mathcal{E})$ the set of firms that have access to market 1 . It is defined according to

$$
F_{1}(\mathcal{E})=\{i \in F \mid(i, 1) \in \mathcal{E}\} .
$$

We first introduce the concept of a contiguous set of firms, which plays a central role in the remainder of the proof. We have the following definition.

Definition C.1 (Contiguous Set). The set $F_{1}(\mathcal{E})$ is contiguous if

$$
F_{1}(\mathcal{E})=\left\{1,2, \ldots,\left|F_{1}(\mathcal{E})\right|\right\}, \quad \text { or } \quad F_{1}(\mathcal{E})=\emptyset .
$$


Here, $\left|F_{1}(\mathcal{E})\right|$ denotes the cardinality of the set $F_{1}(\mathcal{E})$. Qualitatively, the set $F_{1}(\mathcal{E}) \subseteq F$ is contiguous, if it consists of consecutive elements of the set $F$. Clearly, for the edge set $\mathcal{E}^{*}$ generated by the greedy algorithm, the set $F_{1}\left(\mathcal{E}^{*}\right)$ is contiguous.

The rest of the proof consists of two parts. In Part 1, we show that if the set $F_{1}(\mathcal{E})$ is contiguous, then the social welfare at the Nash equilibrium associated with edge set $\mathcal{E}$ is guaranteed to be no larger than that of the edge set $\mathcal{E}^{*}$. In Part 2 , we consider the case in which the set $F_{1}(\mathcal{E})$ is not contiguous. We show that there exists an edge set $\widetilde{\mathcal{E}}$ that yields a contiguous set $F_{1}(\widetilde{\mathcal{E}})$, and has social welfare at Nash equilibrium that is no smaller than that of the edge set $\mathcal{E}$.

Part 1: In this part, we assume that the set $F_{1}(\mathcal{E})$ is contiguous, and show that $\operatorname{SW}\left(q^{\mathrm{NE}}(\mathcal{E}), C\right) \leq$ $\mathrm{SW}\left(q^{\mathrm{NE}}\left(\mathcal{E}^{*}\right), C\right)$. We first define a sequence of edge sets according to

$$
\mathcal{E}_{k}=\bigcup_{i=1}^{k}\{(i, 1)\}, \quad k=0, \ldots, n .
$$

In particular, we have that $\mathcal{E}_{0}=\emptyset$. Let $k^{*}=\left|\mathcal{E}^{*}\right|$. To show that $\operatorname{SW}\left(q^{\mathrm{NE}}(\mathcal{E}), C\right) \leq \operatorname{SW}\left(q^{\mathrm{NE}}\left(\mathcal{E}^{*}\right), C\right)$ if $F_{1}(\mathcal{E})$ is contiguous, it suffices to show that the sequence $\mathrm{SW}\left(q^{\mathrm{NE}}\left(\mathcal{E}_{k}\right), C\right)$ is strictly increasing in $k$ over $k=0, \ldots, k^{*}$, and monotonically non-increasing in $k$ over $k=k^{*}, \ldots, n$.

We assume without loss of generality that $c_{i} \leq \alpha_{1}$ for all $i$. If this is not the case, one can work with an alternative cost function profile $\widetilde{C}=\left(\widetilde{C}_{1}, \ldots, \widetilde{C}_{n}\right)$ that is defined according to

$$
\widetilde{C}_{i}\left(s_{i}\right)=\left(\min \left\{c_{i}, \alpha_{1}\right\} \cdot s_{i}\right)^{+}
$$

for $i=1, \ldots, n$. Clearly, $\min \left\{c_{i}, \alpha_{1}\right\} \leq \alpha_{1}$ for all $i$. Additionally, it is straightforward to show that

$$
\mathrm{SW}\left(q^{\mathrm{NE}}\left(\mathcal{E}_{k}\right), C\right)=\mathrm{SW}\left(q^{\mathrm{NE}}\left(\mathcal{E}_{k}\right), \widetilde{C}\right)
$$

for all $k=0, \ldots, n$.

The proof of this claim on monotonicity relies on the following lemma. Its proof is deferred to Appendix C.3.

Lemma C.1. Assume that each firm $i$ 's cost function is of the form $C_{i}\left(s_{i}\right)=\left(c_{i} s_{i}\right)^{+}$, where $c_{1} \leq \cdots \leq c_{n}$. Let the number of markets $m=1$, and the edge set $\mathcal{E}_{k}$ be defined according to Eq. (19). For each $k=1, \ldots, n$, we have that $\mathrm{SW}\left(q^{\mathrm{NE}}\left(\mathcal{E}_{k}\right), C\right)>\mathrm{SW}\left(q^{\mathrm{NE}}\left(\mathcal{E}_{k-1}\right), C\right)$ if and only if

$$
\alpha_{1}-c_{k}>\frac{1}{k}\left(1+\frac{1}{k-\frac{1}{2(k+1)}}\right)\left(\sum_{i=1}^{k-1}\left(\alpha_{1}-c_{i}\right)\right) \text {. }
$$

It follows from the description of the greedy algorithm that $\mathrm{SW}\left(q^{\mathrm{NE}}\left(\mathcal{E}_{k}\right), C\right)$ is strictly increasing in $k$ for $0 \leq k \leq k^{*}$. Additionally, we have that

$$
\mathrm{SW}\left(q^{\mathrm{NE}}\left(\mathcal{E}_{k^{*}+1}\right), C\right) \leq \mathrm{SW}\left(q^{\mathrm{NE}}\left(\mathcal{E}_{k^{*}}\right), C\right) .
$$

It follows from Lemma C.1 that for $k=k^{*}+1$, the following inequality is satisfied:

$$
\alpha_{1}-c_{k} \leq \frac{1}{k}\left(1+\frac{1}{k-\frac{1}{2(k+1)}}\right)\left(\sum_{i=1}^{k-1}\left(\alpha_{1}-c_{i}\right)\right) .
$$

To complete Part 1 of the proof, we have the following lemma. Its proof can be found in Appendix C.4. 
Lemma C.2. Let $k^{*} \in\{0, \ldots, n\}$, and assume that $c_{1} \leq \cdots \leq c_{n} \leq \alpha_{1}$. If inequality (21) is satisfied for $k=k^{*}+1$, then it is satisfied for $k=k^{*}+1, \ldots, n$.

A combination of Lemma C.1 and C.2 reveals that

$$
\mathrm{SW}\left(q^{\mathrm{NE}}\left(\mathcal{E}_{k}\right), C\right) \leq \mathrm{SW}\left(q^{\mathrm{NE}}\left(\mathcal{E}_{k-1}\right), C\right)
$$

for $k=k^{*}+1, \ldots, n$. This completes Part 1 of the proof.

Part 2: In this part, we assume that the set $F_{1}(\mathcal{E})$ is not contiguous. We show that there exists an edge set $\widetilde{\mathcal{E}} \subseteq F \times\{1\}$, such that the set $F_{1}(\widetilde{\mathcal{E}})$ is contiguous, and $\operatorname{SW}\left(q^{\mathrm{NE}}(\mathcal{E}), C\right) \leq$ $\mathrm{SW}\left(q^{\mathrm{NE}}(\widetilde{\mathcal{E}}), C\right)$.

Our proof of the above claim is constructive. Given an edge set $\mathcal{E} \subseteq F \times\{1\}$, we construct a sequence of $\left(n^{2}+1\right)$ edge sets according to the following procedure

1. Set $k=0$, and $\mathcal{E}_{k}=\mathcal{E}$.

2. If the set $F_{1}\left(\mathcal{E}_{k}\right)$ is contiguous, then set $\mathcal{E}_{k+1}=\mathcal{E}_{k}$, and go to Step 5. If not, go to Step 3 .

3. Define the edge set $\widetilde{\mathcal{E}}_{k}$ according to

$$
\widetilde{\mathcal{E}}_{k}=\mathcal{E}_{k} \backslash\left\{\left(\max F_{1}\left(\mathcal{E}_{k}\right), 1\right)\right\} .
$$

4. If $\mathrm{SW}\left(q^{\mathrm{NE}}\left(\mathcal{E}_{k}\right), C\right) \leq \mathrm{SW}\left(q^{\mathrm{NE}}\left(\widetilde{\mathcal{E}}_{k}\right), C\right)$, then set $\mathcal{E}_{k+1}=\widetilde{\mathcal{E}}_{k}$. If not, set

$$
\mathcal{E}_{k+1}=\widetilde{\mathcal{E}}_{k} \cup\left\{\left(\min \left(F \backslash F_{1}\left(\mathcal{E}_{k}\right)\right), 1\right)\right\} .
$$

5. If $k<n^{2}$, update $k=k+1$, and go to Step 2. If $k \geq n^{2}$, terminate the procedure.

We will show that both of the following claims are true

(i) The set $F_{1}\left(\mathcal{E}_{n^{2}}\right)$ is contiguous.

(ii) For $k=0, \ldots, n^{2}-1$, we have

$$
\mathrm{SW}\left(q^{\mathrm{NE}}\left(\mathcal{E}_{k}\right), C\right) \leq \mathrm{SW}\left(q^{\mathrm{NE}}\left(\mathcal{E}_{k+1}\right), C\right) .
$$

We note that the second claim implies that $\mathrm{SW}\left(q^{\mathrm{NE}}(\mathcal{E}), C\right) \leq \mathrm{SW}\left(q^{\mathrm{NE}}\left(\mathcal{E}_{n^{2}}\right), C\right)$. In what follows, we show that Claim (i) and (ii) are true in Part 2.1 and 2.2 of the proof, respectively.

Part 2.1: Proof of Claim (i). We show that Claim (i) is true according to a "potential function" argument. Namely, we define a potential function on the edge set $\mathcal{E}_{k} \subseteq F \times\{1\}$ as follows

$$
\Phi\left(\mathcal{E}_{k}\right)=\left|F_{1}\left(\mathcal{E}_{k}\right)\right|\left(\max F_{1}\left(\mathcal{E}_{k}\right)-\left|F_{1}\left(\mathcal{E}_{k}\right)\right|\right)
$$

for $k=0, \ldots, n^{2}$. For all $\mathcal{E}_{k} \subseteq F \times\{1\}$, we have that $\Phi\left(\mathcal{E}_{k}\right) \geq 0$. It is straightforward to show $\Phi\left(\mathcal{E}_{k}\right)=0$ if and only if the set $F_{1}\left(\mathcal{E}_{k}\right)$ is contiguous. It follows that if $\Phi\left(\mathcal{E}_{k}\right)>0$, then $\mathcal{E}_{k+1}$ is specified according to either $\mathcal{E}_{k+1}=\widetilde{\mathcal{E}}_{k}$ or Eq. (23). If $\mathcal{E}_{k+1}=\widetilde{\mathcal{E}}_{k}$, we have

$$
\begin{aligned}
& \Phi\left(\mathcal{E}_{k+1}\right)=\left|F_{1}\left(\widetilde{\mathcal{E}}_{k}\right)\right|\left(\max F_{1}\left(\widetilde{\mathcal{E}}_{k}\right)-\left|F_{1}\left(\widetilde{\mathcal{E}}_{k}\right)\right|\right) \\
& \quad=\left|F_{1}\left(\widetilde{\mathcal{E}}_{k}\right)\right|\left(\max F_{1}\left(\widetilde{\mathcal{E}}_{k}\right)-\left(\left|F_{1}\left(\mathcal{E}_{k}\right)\right|-1\right)\right) \\
& \quad \leq\left|F_{1}\left(\widetilde{\mathcal{E}}_{k}\right)\right|\left(\max F_{1}\left(\mathcal{E}_{k}\right)-1-\left(\left|F_{1}\left(\mathcal{E}_{k}\right)\right|-1\right)\right) \\
& \quad=\left(\left|F_{1}\left(\mathcal{E}_{k}\right)\right|-1\right)\left(\max F_{1}\left(\mathcal{E}_{k}\right)-\left|F_{1}\left(\mathcal{E}_{k}\right)\right|\right) \\
& \quad \leq \Phi\left(\mathcal{E}_{k}\right)-1,
\end{aligned}
$$


where inequality (27) follows from the inequality $\max F_{1}\left(\widetilde{\mathcal{E}}_{k}\right) \leq \max F_{1}\left(\mathcal{E}_{k}\right)-1$, Eq. (28) follows from the fact that $\left|F_{1}\left(\widetilde{\mathcal{E}}_{k}\right)\right|=\left|F_{1}\left(\mathcal{E}_{k}\right)\right|-1$, and inequality (29) follows from the fact that $F_{1}\left(\mathcal{E}_{k}\right)$ is not contiguous.

On the other hand, if $\mathcal{E}_{k+1}$ is specified according to Eq. (23), then we have

$$
\begin{aligned}
\Phi\left(\mathcal{E}_{k+1}\right) & =\left|F_{1}\left(\mathcal{E}_{k}\right)\right|\left(\max F_{1}\left(\mathcal{E}_{k+1}\right)-\left|F_{1}\left(\mathcal{E}_{k}\right)\right|\right) \\
& \leq\left|F_{1}\left(\mathcal{E}_{k}\right)\right|\left(\max F_{1}\left(\mathcal{E}_{k}\right)-1-\left|F_{1}\left(\mathcal{E}_{k}\right)\right|\right) \\
& =\Phi\left(\mathcal{E}_{k}\right)-\left|F_{1}\left(\mathcal{E}_{k}\right)\right| \leq \Phi\left(\mathcal{E}_{k}\right)-1,
\end{aligned}
$$

where Eq. (30) follows from the fact that $\left|F_{1}\left(\mathcal{E}_{k}\right)\right|=\left|F_{1}\left(\mathcal{E}_{k+1}\right)\right|$, and inequality (31) follows from the fact that $\max F_{1}\left(\mathcal{E}_{k+1}\right) \leq \max F_{1}\left(\mathcal{E}_{k}\right)-1$. In both cases, we have that if $\Phi\left(\mathcal{E}_{k}\right)>0$, then

$$
\Phi\left(\mathcal{E}_{k}\right)-\Phi\left(\mathcal{E}_{k+1}\right) \geq 1 .
$$

It is straightforward to show that $\Phi\left(\mathcal{E}_{0}\right)<n^{2}$. It immediately follows that $\Phi\left(\mathcal{E}_{n^{2}}\right)=0$. This finishes the proof of Claim (i).

Part 2.2: Proof of Claim (ii). According to the procedure we use in generating the sequence of edge sets, inequality (24) is trivially satisfied if $\mathcal{E}_{k+1}=\mathcal{E}_{k}$ or $\mathcal{E}_{k+1}=\widetilde{\mathcal{E}}_{k}$. For the remainder of this part, we show that inequality (24) is satisfied if $\mathcal{E}_{k+1}$ is specified according to Eq. (23). The key idea in this proof is to show that if the removal of the most expensive producer leads to a strict decrease in social welfare, then a unilateral decrease in the marginal cost of said producer will lead to an increase in social welfare.

We first introduce some notation pertinent to the remainder of the proof. Define the indices $g_{k}$ and $h_{k}$ according to

$$
g_{k}=\max F_{1}\left(\mathcal{E}_{k}\right) \quad \text { and } \quad h_{k}=\min \left(F \backslash F_{1}\left(\mathcal{E}_{k}\right)\right) .
$$

Since the set $F_{1}\left(\mathcal{E}_{k}\right)$ is not contiguous, we must have that $h_{k}<g_{k}$. We define a new cost function profile $C^{\theta}=\left(C_{1}^{\theta}, \ldots, C_{n}^{\theta}\right) \in \mathcal{L}^{n}\left(c_{\min }, c_{\max }\right)$ according to

$$
C_{i}^{\theta}\left(s_{i}\right)= \begin{cases}\left(c_{i} s_{i}\right)^{+} & \text {if } i \neq g_{k} \\ \left(\theta s_{i}\right)^{+} & \text {if } i=g_{k},\end{cases}
$$

where $\theta$ is a scalar parameter.

The unique Nash equillibrium of the networked Cournot game depends on firms' cost function profiles. With a slight abuse of notation, we denote by $q^{\mathrm{NE}}(\mathcal{E}, C)$ the unique Nash equilibrium of the networked Cournot game associated with an edge set $\mathcal{E} \subseteq F \times\{1\}$, and a cost function profile $C$.

Our proof relies on the following technical lemma on the monotonicity of $\mathrm{SW}\left(q^{\mathrm{NE}}\left(\mathcal{E}_{k}, C^{\theta}\right), C^{\theta}\right)$ in the scalar $\theta$. Its proof is deferred to Appendix C.5.

Lemma C.3. Let the edge set $\widetilde{\mathcal{E}}_{k}$ and the cost function profile $C^{\theta}$ be specified according to Eq. (22) and (33), respectively. If $\mathrm{SW}\left(q^{\mathrm{NE}}\left(\mathcal{E}_{k}, C\right), C\right)>\mathrm{SW}\left(q^{\mathrm{NE}}\left(\widetilde{\mathcal{E}}_{k}, C\right), C\right)$, then $\operatorname{SW}\left(q^{\mathrm{NE}}\left(\mathcal{E}_{k}, C^{\theta}\right), C^{\theta}\right)$ is monotonically decreasing in $\theta$ for $c_{\min } \leq \theta \leq c_{g_{k}}$. 
Note that, by specifying $\mathcal{E}_{k+1}$ according to Eq. (23), we essentially replace the most expensive producer with another producer with a cheaper cost. In particular, if $\mathcal{E}_{k+1}$ is specified according to Eq. (23) , then $\mathrm{SW}\left(q^{\mathrm{NE}}\left(\mathcal{E}_{k}, C\right), C\right)$ and $\mathrm{SW}\left(q^{\mathrm{NE}}\left(\mathcal{E}_{k+1}, C\right), C\right)$ are related according to

$$
\begin{aligned}
\operatorname{SW}\left(q^{\mathrm{NE}}\left(\mathcal{E}_{k}, C\right), C\right) & =\operatorname{SW}\left(q^{\mathrm{NE}}\left(\mathcal{E}_{k}, C^{c_{g_{k}}}\right), C^{c_{g_{k}}}\right), \\
\operatorname{SW}\left(q^{\mathrm{NE}}\left(\mathcal{E}_{k+1}, C\right), C\right) & =\operatorname{SW}\left(q^{\mathrm{NE}}\left(\mathcal{E}_{k}, C^{c_{h_{k}}}\right), C^{c_{h_{k}}}\right) .
\end{aligned}
$$

Recall that $h_{k}<g_{k}$. It follows that $c_{\min } \leq c_{h_{k}} \leq c_{g_{k}}$. An application of Lemma C.3 shows that

$$
\mathrm{SW}\left(q^{\mathrm{NE}}\left(\mathcal{E}_{k}, C^{c_{g_{k}}}\right), C^{c_{g_{k}}}\right) \leq \mathrm{SW}\left(q^{\mathrm{NE}}\left(\mathcal{E}_{k}, C^{c_{h_{k}}}\right), C^{c_{h_{k}}}\right) .
$$

The above inequality, in combination with Eq. (34) and (35), shows that when $\mathcal{E}_{k+1}$ is specified according to Eq. (23), inequality (24) is satisfied. This completes the proof.

\section{C.2 Proof of Theorem 5.1}

Without loss of generality, we assume that $c_{1} \leq \cdots \leq c_{n}$. It follows from the description of the greedy algorithm that for each market $j \in M,(1, j) \in \mathcal{E}^{*}$ if and only if $c_{1}<\alpha_{j}$. It immediately follows that the efficient social welfare associated with the edge set $\mathcal{E}^{*}$ satisfies

$$
\mathrm{SW}^{*}\left(\mathcal{E}^{*}, C\right)=\sum_{j=1}^{m} \frac{\left(\left(\alpha_{j}-c_{1}\right)^{+}\right)^{2}}{2 \beta_{j}}=\mathrm{SW}^{*}(F \times M, C) .
$$

For the second part of the theorem, we only provide a proof for the case in which the number of markets $m=1$. The generalization to the case in which $m>1$ can be carried out following similar steps as in the proof of Proposition 3.2 .

Given the restriction that $c_{1} \leq \cdots \leq c_{n}$, we define the subset of ordered linear cost function profiles in $\mathcal{L}^{n}\left(c_{\min }, c_{\max }\right)$ according to

$$
\begin{aligned}
& \mathcal{O}^{n}\left(c_{\min }, c_{\max }\right)=\left\{C \in \mathcal{L}^{n}\left(c_{\min }, c_{\max }\right) \mid\right. \\
&\left.C_{i}\left(s_{i}\right)=\left(c_{i} s_{i}\right)^{+}, i=1, \ldots, n, c_{1} \leq \cdots \leq c_{n}\right\} .
\end{aligned}
$$

Thus, we have the following chain of inequalities

$$
\begin{aligned}
& \rho\left(\mathcal{E}^{*}, C\right)=\inf _{\mathcal{E} \subseteq F \times M} \frac{\mathrm{SW}^{*}(F \times M, C)}{\operatorname{SW}\left(q^{\mathrm{NE}}(\mathcal{E}), C\right)} \\
& \leq \sup _{C \in \mathcal{L}^{n}\left(c_{\min }, c_{\max }\right)} \inf _{\mathcal{E} \subseteq F \times M} \frac{\mathrm{SW}^{*}(F \times M, C)}{\mathrm{SW}\left(q^{\mathrm{NE}}(\mathcal{E}), C\right)} \\
& =\sup _{C \in \mathcal{O}^{n}\left(c_{\text {min }}, c_{\text {max }}\right)} \inf _{\{(1,1)\} \subseteq \mathcal{E} \subseteq F \times M} \frac{\mathrm{SW}^{*}(F \times M, C)}{\mathrm{SW}\left(q^{\mathrm{NE}}(\mathcal{E}), C\right)} \\
& \leq_{\{(1,1)\} \subseteq \mathcal{E} \subseteq F \times M} \sup _{C \in \mathcal{O}^{n}\left(c_{\min }, c_{\max }\right)} \frac{\mathrm{SW}^{*}(F \times M, C)}{\mathrm{SW}\left(q^{\mathrm{NE}}(\mathcal{E}), C\right)} \\
& \leq \inf _{\{(1,1)\} \subseteq \mathcal{E} \subseteq F \times M} \frac{1}{\frac{2|\mathcal{E}|+4}{3|\mathcal{E}+5|}+\delta\left(\gamma_{1},|\mathcal{E}|\right)} \\
& =\frac{1}{\max _{k \in\{1, \ldots, n\}}\left\{\frac{2 k+4}{3 k+5}+\delta\left(\gamma_{1}, k\right)\right\}} \text {. }
\end{aligned}
$$


Here, Eq. (36) follows from the fact that $\mathrm{SW}^{*}\left(\mathcal{E}^{*}, C\right)=\mathrm{SW}^{*}(F \times M, C)$, Eq. (38) follows from our restriction that $c_{1} \leq \cdots \leq c_{n}$, inequality (39) follows from the min-max inequality, and inequality (40) is a direct application of Proposition 3.2. This completes the proof.

\section{C.3 Proof of Lemma C.1}

The proof proceeds in two parts. In Part 1, we provide a necessary and sufficient condition for the production quantity of firm $k$ to be strictly positive at Nash equilibrium, when the edge set is given by $\mathcal{E}_{k}$. In Part 2 , we leverage on the intermediary result in Part 1 to show that $\mathrm{SW}\left(q^{\mathrm{NE}}\left(\mathcal{E}_{k}\right), C\right)>$ $\mathrm{SW}\left(q^{\mathrm{NE}}\left(\mathcal{E}_{k-1}\right), C\right)$ if and only if inequality (20) is satisfied.

Part 1: We show that for the game $\left(F, \mathcal{Q}\left(\mathcal{E}_{k}\right), \pi\right)$, the production quantity of firm $k$ at Nash equilibrium $q_{k 1}^{\mathrm{NE}}\left(\mathcal{E}_{k}\right)$ is strictly positive, if and only if

$$
\alpha_{1}-c_{k}>\frac{1}{k}\left(\sum_{i=1}^{k-1}\left(\alpha_{1}-c_{i}\right)\right)
$$

We first prove the "if" part of the desired claim. First note that inequality (42) implies that the following inequality is satisfied:

$$
(k+1)\left(\alpha_{1}-c_{k}\right)>\sum_{i=1}^{k}\left(\alpha_{1}-c_{i}\right) .
$$

One can check that if inequality (42) is satisfied, then the unique Nash equilibrium of the game $\left(F, \mathcal{Q}\left(\mathcal{E}_{k}\right), \pi\right)$ is given by

$$
q_{i 1}^{\mathrm{NE}}\left(\mathcal{E}_{k}\right)=\frac{(k+1)\left(\alpha_{1}-c_{i}\right)-\sum_{\ell=1}^{k}\left(\alpha_{1}-c_{\ell}\right)}{(k+1) \beta_{1}}, i=1, \ldots, k,
$$

and $q_{i 1}^{\mathrm{NE}}\left(\mathcal{E}_{k}\right)=0$ for $i=k+1, \ldots, n$. It follows from inequality (43) that $q_{k 1}^{\mathrm{NE}}\left(\mathcal{E}_{k}\right)>0$.

Next, we prove the "only if" part of the desired claim. First note that $c_{i} \leq c_{k}$ for $i=1, \ldots, k$. Recall that for the game $\left(F, \mathcal{Q}\left(\mathcal{E}_{k}\right), \pi\right)$, firm $k$ 's production quantity $q_{k 1}^{\mathrm{NE}}\left(\mathcal{E}_{k}\right)$ at Nash equilibrium is strictly positive. It follows that $q_{i 1}^{\mathrm{NE}}\left(\mathcal{E}_{k}\right)>0$ for $i=1, \ldots, k$. The first order optimality condition for Nash equilibrium of the game $\left(F, \mathcal{Q}\left(\mathcal{E}_{k}\right), \pi\right)$ implies that

$$
\alpha_{1}-\beta_{1}\left(\sum_{\ell=1}^{k} q_{\ell 1}^{\mathrm{NE}}\left(\mathcal{E}_{k}\right)\right)-\beta_{1} q_{i 1}^{\mathrm{NE}}\left(\mathcal{E}_{k}\right)-c_{i}=0,
$$

for $i=1, \ldots, k$. Consequently, we have that

$$
q_{k 1}^{\mathrm{NE}}\left(\mathcal{E}_{k}\right)=\frac{(k+1)\left(\alpha_{1}-c_{k}\right)-\sum_{\ell=1}^{k}\left(\alpha_{1}-c_{\ell}\right)}{(k+1) \beta_{1}}>0 .
$$

This implies that inequality (42) is satisfied.

Part 2: We show SW $\left(q^{\mathrm{NE}}\left(\mathcal{E}_{k}\right), C\right)>\mathrm{SW}\left(q^{\mathrm{NE}}\left(\mathcal{E}_{k-1}\right), C\right)$ if and only if inequality (20) is satisfied. First note when $k=1$, it is straightforward to see that $\mathrm{SW}\left(q^{\mathrm{NE}}\left(\mathcal{E}_{1}\right), C\right)>0$ if and only if $\alpha_{1}-c_{1}>0$. Thus, for the remainder of the proof, we assume that $k \geq 2$. 
We only provide a proof for the "only if" part of the claim, as the "if" part of the claim can be proved using similar arguments. First note that $\mathrm{SW}\left(q^{\mathrm{NE}}\left(\mathcal{E}_{k}\right), C\right)>\mathrm{SW}\left(q^{\mathrm{NE}}\left(\mathcal{E}_{k-1}\right), C\right)$ implies that $q_{k 1}^{\mathrm{NE}}\left(\mathcal{E}_{k}\right)>0$. If this is not the case, then we have that $q^{\mathrm{NE}}\left(\mathcal{E}_{k}\right)=q^{\mathrm{NE}}\left(\mathcal{E}_{k-1}\right)$, which clearly leads to a contradiction. Note that $c_{i} \leq c_{k}$ for $i=1, \ldots, k$. It follows that

$$
q_{i 1}^{\mathrm{NE}}\left(\mathcal{E}_{k}\right) \geq q_{k 1}^{\mathrm{NE}}\left(\mathcal{E}_{k}\right)>0, \quad \text { for } \quad i=1, \ldots, k .
$$

One can show that the unique Nash equilibrium of the game $\left(F, \mathcal{Q}\left(\mathcal{E}_{k}\right), \pi\right)$ is given by

$$
q_{i 1}^{\mathrm{NE}}\left(\mathcal{E}_{k}\right)=\frac{(k+1)\left(\alpha_{1}-c_{i}\right)-\sum_{\ell=1}^{k}\left(\alpha_{1}-c_{\ell}\right)}{(k+1) \beta_{1}}, i=1, \ldots, k,
$$

and $q_{i 1}^{\mathrm{NE}}\left(\mathcal{E}_{k}\right)=0$ for $i=k+1, \ldots, n$. We denote the aggregate supply in market 1 at the unique Nash equilibrium of the game $\left(F, \mathcal{Q}\left(\mathcal{E}_{k}\right), \pi\right)$ by $d_{1}^{\mathrm{NE}}\left(\mathcal{E}_{k}\right)$. It is given by

$$
d_{1}^{\mathrm{NE}}\left(\mathcal{E}_{k}\right)=\sum_{i=1}^{n} q_{i 1}^{\mathrm{NE}}\left(\mathcal{E}_{k}\right)=\frac{\sum_{i=1}^{k}\left(\alpha_{1}-c_{i}\right)}{(k+1) \beta_{1}}
$$

Additionally, the social welfare at the Nash equilibrium of the game $\left(F, \mathcal{Q}\left(\mathcal{E}_{k}\right), \pi\right)$ satisfies

$$
\begin{aligned}
& \operatorname{SW}\left(q^{\mathrm{NE}}\left(\mathcal{E}_{k}\right), C\right) \\
= & \alpha_{1} d_{1}^{\mathrm{NE}}\left(\mathcal{E}_{k}\right)-\frac{1}{2} \beta_{1} d_{1}^{\mathrm{NE}^{2}}\left(\mathcal{E}_{k}\right)-\sum_{i=1}^{k} c_{i} q_{i 1}^{\mathrm{NE}}\left(\mathcal{E}_{k}\right) \\
= & \alpha_{1} d_{1}^{\mathrm{NE}}\left(\mathcal{E}_{k}\right)-\frac{1}{2} \beta_{1} d_{1}^{\mathrm{NE}^{2}}\left(\mathcal{E}_{k}\right)-\sum_{i=1}^{k} c_{i}\left(\frac{\alpha_{1}-c_{i}}{\beta_{1}}-d_{1}^{\mathrm{NE}}\left(\mathcal{E}_{k}\right)\right) \\
= & (k+1) \alpha_{1} d_{1}^{\mathrm{NE}}\left(\mathcal{E}_{k}\right)-\frac{1}{2} \beta_{1} d_{1}^{\mathrm{NE}}{ }^{2}\left(\mathcal{E}_{k}\right)-\sum_{i=1}^{k}\left(\frac{c_{i}\left(\alpha_{1}-c_{i}\right)}{\beta_{1}}\right)+\left(-k \alpha_{1}+\sum_{i=1} c_{i}\right) d_{1}^{\mathrm{NE}}\left(\mathcal{E}_{k}\right) \\
= & \frac{\alpha_{1} \sum_{i=1}^{k}\left(\alpha_{1}-c_{i}\right)}{\beta_{1}}-\frac{2 k+3}{2} \beta_{1} d_{1}^{\mathrm{NE}}\left(\mathcal{E}_{k}\right)-\sum_{i=1}^{k} \frac{c_{i}\left(\alpha_{1}-c_{i}\right)}{\beta_{1}} \\
= & \frac{\sum_{i=1}^{k}\left(\alpha_{1}-c_{i}\right)^{2}}{\beta_{1}}-\frac{2 k+3}{2} \beta_{1} d_{1}^{\mathrm{NE}}\left(\mathcal{E}_{k}\right) \\
= & \frac{\sum_{i=1}^{k}\left(\alpha_{1}-c_{i}\right)^{2}}{\beta_{1}}-\frac{2 k+3}{2} \frac{\left(\sum_{i=1}^{k}\left(\alpha_{1}-c_{i}\right)\right)^{2}}{(k+1)^{2} \beta_{1}} .
\end{aligned}
$$

Next, we provide a closed-form expression for $\mathrm{SW}\left(q^{\mathrm{NE}}\left(\mathcal{E}_{k-1}\right), C\right)$. Recall that $q_{k 1}^{\mathrm{NE}}\left(\mathcal{E}_{k}\right)>0$. As we showed in Part 1, this implies that inequality (42) is satisfied. We thus have

$$
\alpha_{1}-c_{k-1} \geq \alpha_{1}-c_{k}>\frac{1}{k}\left(\sum_{i=1}^{k-1}\left(\alpha_{1}-c_{i}\right)\right),
$$

which further implies that

$$
\alpha_{1}-c_{k-1}>\frac{1}{k-1}\left(\sum_{i=1}^{k-2}\left(\alpha_{1}-c_{i}\right)\right)
$$


It follows from our result in Part 1 that for the game $\left(F, \mathcal{Q}\left(\mathcal{E}_{k-1}\right), \pi\right)$, producer $k-1$ 's production quantity at Nash equilibrium is strictly positive. Using similar arguments as in our derivation on the closed-form expression for $\mathrm{SW}\left(q^{\mathrm{NE}}\left(\mathcal{E}_{k}\right), C\right)$, we have the following closed-form expression for $\mathrm{SW}\left(q^{\mathrm{NE}}\left(\mathcal{E}_{k-1}\right), C\right)$

$$
\begin{aligned}
\mathrm{SW} & \left(q^{\mathrm{NE}}\left(\mathcal{E}_{k-1}\right), C\right) \\
& =\frac{\sum_{i=1}^{k-1}\left(\alpha_{1}-c_{i}\right)^{2}}{\beta_{1}}-\frac{2 k+1}{2} \frac{\left(\sum_{i=1}^{k-1}\left(\alpha_{1}-c_{i}\right)\right)^{2}}{k^{2} \beta_{1}} .
\end{aligned}
$$

Thus, the difference between $\mathrm{SW}\left(q^{\mathrm{NE}}\left(\mathcal{E}_{k}\right), C\right)$ and $\mathrm{SW}\left(q^{\mathrm{NE}}\left(\mathcal{E}_{k-1}\right), C\right)$ is given by

$$
\begin{aligned}
\mathrm{SW}\left(q^{\mathrm{NE}}\left(\mathcal{E}_{k}\right), C\right)-\mathrm{SW}\left(q^{\mathrm{NE}}\left(\mathcal{E}_{k-1}\right), C\right) \\
=\frac{\left(\alpha_{1}-c_{k}\right)^{2}}{\beta_{1}}+\frac{2 k+1}{2} \frac{\left(\sum_{i=1}^{k-1}\left(\alpha_{1}-c_{i}\right)\right)^{2}}{k^{2} \beta_{1}} \\
\quad-\frac{2 k+3}{2} \frac{\left(\sum_{i=1}^{k}\left(\alpha_{1}-c_{i}\right)\right)^{2}}{(k+1)^{2} \beta_{1}} .
\end{aligned}
$$

An algebraic calculation reveals that $\mathrm{SW}\left(q^{\mathrm{NE}}\left(\mathcal{E}_{k}\right), C\right)>\mathrm{SW}\left(q^{\mathrm{NE}}\left(\mathcal{E}_{k-1}\right), C\right)$ if and only if

$$
\frac{\alpha_{1}-c_{k}}{\sum_{i=1}^{k-1}\left(\alpha_{1}-c_{i}\right)}<\frac{1}{k}, \quad \text { or } \quad \frac{\alpha_{1}-c_{k}}{\sum_{i=1}^{k-1}\left(\alpha_{1}-c_{i}\right)}>\frac{k+2+\frac{1}{2 k}}{k^{2}+k-\frac{1}{2}} .
$$

Recall that $\mathrm{SW}\left(q^{\mathrm{NE}}\left(\mathcal{E}_{k}\right), C\right)>\mathrm{SW}\left(q^{\mathrm{NE}}\left(\mathcal{E}_{k-1}\right), C\right)$ implies that inequality (42) is satisfied. It follows from the above inequality that $\mathrm{SW}\left(q^{\mathrm{NE}}\left(\mathcal{E}_{k}\right), C\right)>\operatorname{SW}\left(q^{\mathrm{NE}}\left(\mathcal{E}_{k-1}\right), C\right)$ implies inequality (20) is satisfied.

\section{C.4 Proof of Lemma C.2}

We prove this lemma by induction in $k$.

Base Step: For $k=k^{*}+1$, inequality (21) is satisfied by the assumption of this lemma.

Induction Step: Assume that inequality (21) is satisfied for $k \geq 1$. We show that it is satisfied for $k+1$ by showing that

$$
\alpha_{1}-c_{k+1} \leq \frac{1}{k+1}\left(1+\frac{1}{k+1-\frac{1}{2(k+2)}}\right)\left(\sum_{i=1}^{k}\left(\alpha_{1}-c_{i}\right)\right) .
$$

Since $c_{k+1} \geq c_{k}$, inequality (45) is satisfied if the following inequality holds

$$
(k+1)\left(\alpha_{1}-c_{k}\right) \leq\left(1+\frac{1}{k+1-\frac{1}{2(k+2)}}\right)\left(\sum_{i=1}^{k}\left(\alpha_{1}-c_{i}\right)\right) .
$$

An algebraic calculation reveals that inequality (46) is satisfied if and only if

$$
\frac{k^{3}+3 k^{2}+\frac{1}{2} k-2}{k^{2}+3 k+\frac{3}{2}}\left(\alpha_{1}-c_{k}\right) \leq \frac{k^{2}+4 k+\frac{7}{2}}{k^{2}+3 k+\frac{3}{2}}\left(\sum_{i=1}^{k-1}\left(\alpha_{1}-c_{i}\right)\right) .
$$


Given that $k \geq 1$, the above inequality is satisfied if and only if

$$
\alpha_{1}-c_{k} \leq \frac{k^{2}+4 k+\frac{7}{2}}{k^{3}+3 k^{2}+\frac{1}{2} k-2}\left(\sum_{i=1}^{k-1}\left(\alpha_{1}-c_{i}\right)\right) .
$$

The induction hypothesis implies that inequality (21) is satisfied for $k$. Given that $c_{i} \leq \alpha_{1}$ for $i=1, \ldots, n$, we have that inequality (47) is satisfied if

$$
\frac{1}{k}\left(1+\frac{1}{k-\frac{1}{2(k+1)}}\right) \leq \frac{k^{2}+4 k+\frac{7}{2}}{k^{3}+3 k^{2}+\frac{1}{2} k-2} .
$$

Given that $k \geq 1$, inequality (48) holds if and only if

$$
\begin{gathered}
\left(k^{2}+2 k+\frac{1}{2}\right)\left(k^{3}+3 k^{2}+\frac{1}{2} k-2\right) \\
\leq k\left(k^{2}+k-\frac{1}{2}\right)\left(k^{2}+4 k+\frac{7}{2}\right) .
\end{gathered}
$$

And the above inequality holds if and only if $(k+1)^{2} \geq 0$. Thus, (48) is satisfied if $k \geq 1$. This further implies that inequalities (45)-(47) are all satisfied. Hence, inequality (21) also holds for $k+1$. This completes the proof by induction.

\section{C.5 Proof of Lemma C.3}

Let $n_{k}=\left|F_{1}\left(\mathcal{E}_{k}\right)\right|$. For the ease of exposition, we assume that the set $F_{1}\left(\mathcal{E}_{k}\right)$ is given by

$$
F_{1}\left(\mathcal{E}_{k}\right)=\left\{1,2, \ldots, n_{k}-1, g_{k}\right\}
$$

However, it is straightforward to generalize the proof to the case in which $F_{1}\left(\mathcal{E}_{k}\right)$ is any subset of $\left\{1,2, \ldots, g_{k}\right\}$ satisfying $n_{k}=\left|F_{1}\left(\mathcal{E}_{k}\right)\right|$ and $\max F_{1}\left(\mathcal{E}_{k}\right)=g_{k}$.

The remainder proof proceeds in two parts. In the first part, we provide a closed-form expression for $\mathrm{SW}\left(q^{\mathrm{NE}}\left(\mathcal{E}_{k}, C^{\theta}\right), C^{\theta}\right)$, and show that it is piecewise quadratic in $\theta$. In Part 2 , we show that given the assumption stated in this lemma, $\mathrm{SW}\left(q^{\mathrm{NE}}\left(\mathcal{E}_{k}, C^{\theta}\right), C^{\theta}\right)$ is strictly decreasing in $\theta$ for $c_{\min } \leq \theta \leq c_{g_{k}}$.

Part 1: We first show that when $\theta=c_{g_{k}}$, we have that $q_{i 1}^{\mathrm{NE}}\left(\mathcal{E}, C^{\theta}\right)>0$ for all $i \in F_{1}\left(\mathcal{E}_{k}\right)$. First recall that

$$
\operatorname{SW}\left(q^{\mathrm{NE}}\left(\mathcal{E}_{k}, C\right), C\right)>\operatorname{SW}\left(q^{\mathrm{NE}}\left(\widetilde{\mathcal{E}}_{k}, C\right), C\right) .
$$

It follows that $q_{g_{k} 1}^{\mathrm{NE}}\left(\mathcal{E}_{k}, C\right)>0$. If this is not the case, then Nash equilibrium remains unchanged after the removal of producer $g_{k}$. This implies that $\mathrm{SW}\left(q^{\mathrm{NE}}\left(\mathcal{E}_{k}, C\right), C\right)=\operatorname{SW}\left(q^{\mathrm{NE}}\left(\widetilde{\mathcal{E}}_{k}, C\right), C\right)$, which is a contradiction. Recall that $c_{i} \leq c_{g_{k}}$ for all $i \in F_{1}\left(\mathcal{E}_{k}\right)$. In combination with the fact that $q_{g_{k} 1}^{\mathrm{NE}}\left(\mathcal{E}_{k}, C\right)>0$, this implies that $q_{i 1}^{\mathrm{NE}}\left(\mathcal{E}, C^{\theta}\right)>0$ for all $i \in F_{1}\left(\mathcal{E}_{k}\right)$.

Assume that when $\theta=c_{\min }$, the vector $q^{\mathrm{NE}}\left(\mathcal{E}_{k}, C^{\theta}\right)$ includes $n_{\min }$ strictly positive entries. Since $\left|\mathcal{E}_{k}\right|=n_{k}$, we must have that $n_{\text {min }} \leq n_{k}$. We define a collection of subsets $\Theta_{n_{\min }}, \ldots, \Theta_{n_{k}}$ of the set 
$\left[c_{\min }, c_{g_{k}}\right]$ according to

$$
\begin{aligned}
\Theta_{\ell}= & {\left[\alpha_{1}+\sum_{r=1}^{\ell-2}\left(\alpha_{1}-c_{r}\right)-\ell\left(\alpha_{1}-c_{\ell-1}\right),\right.} \\
& \left.\alpha_{1}+\sum_{r=1}^{\ell-1}\left(\alpha_{1}-c_{r}\right)-(\ell+1)\left(\alpha_{1}-c_{\ell}\right)\right) \bigcap\left[c_{\min }, c_{g_{k}}\right],
\end{aligned}
$$

for $\ell=n_{\min }, \ldots, n_{k}-1$, and

$$
\Theta_{n_{k}}=\left[c_{\min }, c_{g_{k}}\right] \backslash \bigcup_{\ell=n_{\min }}^{n_{k}-1} \Theta_{\ell} .
$$

One can check that for any $\theta \in \operatorname{int}\left(\Theta_{\ell}\right), \ell \in\left\{n_{\min }, \ldots, n_{k}\right\}$, we have

$$
q_{i 1}^{\mathrm{NE}}\left(\mathcal{E}_{k}, C^{\theta}\right)>0, \text { for } i=1, \ldots, \ell-1, \text { and } i=g_{k} .
$$

That is, the vector $q^{\mathrm{NE}}\left(\mathcal{E}_{k}, C^{\theta}\right)$ contains $\ell$ strictly positive entries. Additionally, given that the vector $q^{\mathrm{NE}}\left(\mathcal{E}_{k}, C^{\theta}\right)$ includes $n_{\min }$ strictly positive entries when $\theta=c_{\min }$, we can show that

$$
\bigcup_{\ell=n_{\min }}^{n_{k}} \Theta_{\ell}=\left[c_{\min }, c_{g_{k}}\right], \quad \text { and } \quad \Theta_{\ell_{1}} \bigcap \Theta_{\ell_{2}}=\emptyset
$$

for any $\ell_{1}, \ell_{2} \in\left\{n_{\min }, \ldots, n_{k}\right\}$ satisfying $\ell_{1} \neq \ell_{2}, 1$

If follows from similar arguments as in the proof of Lemma C.1 that $\mathrm{SW}\left(q^{\mathrm{NE}}\left(\mathcal{E}_{k}, C^{\theta}\right), C^{\theta}\right)$ admits the following closed-form expression

$$
\begin{aligned}
\mathrm{SW} & \left(q^{\mathrm{NE}}\left(\mathcal{E}_{k}, C^{\theta}\right), C^{\theta}\right)=\frac{\left(\alpha_{1}-\theta\right)^{2}+\sum_{r=1}^{\ell-1}\left(\alpha_{1}-c_{r}\right)^{2}}{\beta_{1}} \\
& -\frac{2 \ell+3}{2} \frac{\left(\alpha_{1}-\theta+\sum_{r=1}^{\ell-1}\left(\alpha_{1}-c_{r}\right)\right)^{2}}{(\ell+1)^{2} \beta_{1}}, \text { for } \theta \in \Theta_{\ell} .
\end{aligned}
$$

We remark that $\mathrm{SW}\left(q^{\mathrm{NE}}\left(\mathcal{E}_{k}, C^{\theta}\right), C^{\theta}\right)$ is a piecewise quadratic function of $\theta$ that is continuous in $\theta$ for $\theta \in\left[c_{\min }, c_{g_{k}}\right]$, and continuously differentiable in $\theta$ for $\theta \in \operatorname{int}\left(\Theta_{\ell}\right), \ell \in\left\{n_{\min }, \ldots, n_{k}\right\}$.

Part 2: We show that $\mathrm{SW}\left(q^{\mathrm{NE}}\left(\mathcal{E}_{k}, C^{\theta}\right), C^{\theta}\right)$ is strictly monotonically decreasing in $\theta$ for $\theta \in$ $\left[c_{\min }, c_{g_{k}}\right]$. First recall that the union of the intervals $\Theta_{n_{\min }}, \ldots, \Theta_{n_{k}}$ satisfies

$$
\bigcup_{\ell=n_{\min }}^{n_{k}} \Theta_{\ell}=\left[c_{\min }, c_{g_{k}}\right]
$$

Additionally, SW $\left(q^{\mathrm{NE}}\left(\mathcal{E}_{k}\right), C^{\theta}\right)$ is continuous in $\theta$ for $\theta \in\left[c_{\min }, c_{g_{k}}\right]$. Thus, in order to show that social welfare $\mathrm{SW}\left(q^{\mathrm{NE}}\left(\mathcal{E}_{k}\right), C^{\theta}\right)$ is strictly decreasing in $\theta$ on $\left[c_{\mathrm{min}}, c_{g_{k}}\right]$, it suffices to show that

$$
\frac{\partial}{\partial \theta} \mathrm{SW}\left(q^{\mathrm{NE}}\left(\mathcal{E}_{k}\right), C^{\theta}\right)<0
$$

\footnotetext{
${ }^{1}$ The collection of sets $\left\{\Theta_{n_{\min }}, \ldots, \Theta_{n_{k}}\right\}$ might not be a partition of $\left[c_{\min }, c_{g_{k}}\right]$, as some of these sets can be empty.
} 
for $\theta \in \operatorname{int}\left(\Theta_{\ell}\right), \ell=n_{\min }, \ldots, n_{k}$.

For $\theta \in \operatorname{int}\left(\Theta_{\ell}\right)$, we have the following closed-form expression for $\partial \mathrm{SW}\left(q^{\mathrm{NE}}\left(\mathcal{E}_{k}\right), C^{\theta}\right) / \partial \theta$ :

$$
\begin{aligned}
\frac{\partial}{\partial \theta} \mathrm{SW} & \left(q^{\mathrm{NE}}\left(\mathcal{E}_{k}\right), C^{\theta}\right) \\
= & \frac{(2 \ell+3) \sum_{r=1}^{\ell-1}\left(\alpha_{1}-c_{r}\right)-\left(2 \ell^{2}+2 \ell-1\right)\left(\alpha_{1}-\theta\right)}{(\ell+1)^{2} \beta_{1}}
\end{aligned}
$$

Thus, for each $\theta \in \operatorname{int}\left(\Theta_{\ell}\right), \partial \mathrm{SW}\left(q^{\mathrm{NE}}\left(\mathcal{E}_{k}\right), C^{\theta}\right) / \partial \theta<0$ if the following inequality is satisfied:

$$
\alpha_{1}-\theta>\frac{2 \ell+3}{2 \ell^{2}+2 \ell-1} \sum_{r=1}^{\ell-1}\left(\alpha_{1}-c_{r}\right) .
$$

We first show that $\partial \mathrm{SW}\left(q^{\mathrm{NE}}\left(\mathcal{E}_{k}\right), C^{\theta}\right) / \partial \theta<0$ for $\theta \in \operatorname{int}\left(\Theta_{n_{k}}\right)$. Recall that we have that $\mathrm{SW}\left(q^{\mathrm{NE}}\left(\mathcal{E}_{k}, C\right), C\right)>\operatorname{SW}\left(q^{\mathrm{NE}}\left(\widetilde{\mathcal{E}}_{k}, C\right), C\right)$. It follows from Lemma C.1 that the following inequality is satisfied

$$
\alpha_{1}-c_{g_{k}}>\frac{1}{n_{k}}\left(1+\frac{1}{n_{k}-\frac{1}{2\left(n_{k}+1\right)}}\right) \sum_{r=1}^{n_{k}-1}\left(\alpha_{1}-c_{r}\right) .
$$

It follows from inequality (50) that for each $\theta \in \operatorname{int}\left(\Theta_{n_{k}}\right)$, we have

$$
\begin{aligned}
\alpha_{1}-\theta & >\alpha_{1}-c_{g_{k}}>\frac{1}{n_{k}}\left(1+\frac{1}{n_{k}-\frac{1}{2\left(n_{k}+1\right)}}\right) \sum_{r=1}^{n_{k}-1}\left(\alpha_{1}-c_{r}\right) \\
& >\frac{2 n_{k}+3}{2 n_{k}^{2}+2 n_{k}-1} \sum_{r=1}^{n_{k}-1}\left(\alpha_{1}-c_{r}\right) .
\end{aligned}
$$

Thus, $\partial \mathrm{SW}\left(q^{\mathrm{NE}}\left(\mathcal{E}_{k}\right), C^{\theta}\right) / \partial \theta<0$ for $\theta \in \operatorname{int}\left(\Theta_{n_{k}}\right)$.

Next, we show that $\partial \mathrm{SW}\left(q^{\mathrm{NE}}\left(\mathcal{E}_{k}\right), C^{\theta}\right) / \partial \theta<0$ for $\theta \in \operatorname{int}\left(\Theta_{\ell}\right), \ell=n_{\min }, \ldots, n_{k}-1$. Recall that $c_{1} \leq \cdots \leq c_{n_{k}-1} \leq c_{g_{k}}<\alpha_{1}$. It follows from a combination of Lemma C.2 and inequality (50) that

$$
\alpha_{1}-c_{\ell}>\frac{1}{\ell}\left(1+\frac{1}{\ell-\frac{1}{2(\ell+1)}}\right) \sum_{r=1}^{\ell-1}\left(\alpha_{1}-c_{r}\right)
$$

for $\ell=n_{\min }, \ldots, n_{k}-1$. Inequality (51) implies that

$$
\begin{aligned}
\ell\left(\alpha_{1}-c_{\ell}\right) & >\left(1+\frac{1}{\ell-\frac{1}{2(\ell+1)}}\right) \sum_{r=1}^{\ell-1}\left(\alpha_{1}-c_{r}\right) \\
& >\sum_{r=1}^{\ell-1}\left(\alpha_{1}-c_{r}\right)
\end{aligned}
$$


for $\ell=n_{\min }, \ldots, n_{k}-1$. It follows from inequality (52) that the following chain of inequalities are satisfied for $\theta \in \operatorname{int}\left(\Theta_{\ell}\right), \ell=n_{\min }, \ldots, n_{k}-1$ :

$$
\begin{aligned}
\theta & <\alpha_{1}+\sum_{r=1}^{\ell-1}\left(\alpha_{1}-c_{r}\right)-(\ell+1)\left(\alpha_{1}-c_{\ell}\right) \\
& =c_{\ell}+\sum_{r=1}^{\ell-1}\left(\alpha_{1}-c_{r}\right)-\ell\left(\alpha_{1}-c_{\ell}\right)<c_{\ell} .
\end{aligned}
$$

The above inequality, in combination with inequality (51), provides the following lower bound on $\alpha_{1}-\theta$ for $\theta \in \operatorname{int}\left(\Theta_{\ell}\right)$ :

$$
\begin{gathered}
\alpha_{1}-\theta>\alpha_{1}-c_{\ell}>\frac{1}{\ell}\left(1+\frac{1}{\ell-\frac{1}{2(\ell+1)}}\right) \sum_{r=1}^{\ell-1}\left(\alpha_{1}-c_{r}\right) \\
>\frac{2 \ell+3}{2 \ell^{2}+2 \ell-1} \sum_{r=1}^{\ell-1}\left(\alpha_{1}-c_{r}\right) .
\end{gathered}
$$

Thus, $\partial \mathrm{SW}\left(q^{\mathrm{NE}}\left(\mathcal{E}_{k}\right), C^{\theta}\right) / \partial \theta<0$ for $\theta \in \operatorname{int}\left(\Theta_{\ell}\right), \ell=n_{\min }, \ldots, n_{k}-1$. This completes the proof.

\section{Proofs from Section 6}

Proof. Proof of Theorem 6.1

Example D.1. In the case of symmetric linear costs from the previous section, recall that consumer surplus can be written:

$$
C S_{j}^{N E}=\frac{\left(\alpha_{j}-c_{1}\right)^{2} n^{2}}{2 \beta_{j}(n+1)^{2}}
$$

and in this case, the overall penalty with $n$ firms can be written:

$$
p_{j}=\theta \frac{\left(\alpha_{j}-c_{1}\right)^{2} n^{2}}{2 \beta_{j}(n+1)^{2}} f(n), 0 \leq \theta \leq 1
$$

In this scenario, we can again make the (tight) price of anarchy explicit for the case with search costs where $\max _{j} \alpha_{j}>c$, i.e. when the solution is non-degenerate:

$$
\rho(F \times M, \bar{C}) \leq \frac{(n+1)^{2}}{n^{2}(1-\theta f(n))+2 n}
$$

We can already see in the above example that search costs can bring significant changes to the price of anarchy bounds we obtain. For example, when $\theta=0$, then we obtain the original bound for open access with symmetric costs firms, suggesting that the larger $n$ is, the better the bound. On the other hand, when $\theta=1$, as $n$ grows large, $\theta f(n) \rightarrow 1$, and our price of anarchy bound becomes linear in $n$, the number of firms. 